\title{
Heterogeneous fibroblasts underlie age-dependent tertiary lymphoid tissues in the kidney
}

Yuki Sato, ${ }^{1}$ Akiko Mii, ${ }^{1}$ Yoko Hamazaki, ${ }^{2}$ Harumi Fujita, ${ }^{2}$ Hirosuke Nakata, ${ }^{1}$ Kyoko Masuda, ${ }^{3}$ Shingo Nishiyama, ${ }^{1}$ Shinsuke Shibuya, ${ }^{4}$ Hironori Haga, ${ }^{4}$ Osamu Ogawa, ${ }^{5}$ Akira Shimizu, ${ }^{6}$ Shuh Narumiya, ${ }^{7}$ Tsuneyasu Kaisho, ${ }^{8}$ Makoto Arita, ${ }^{9}$ Masashi Yanagisawa, ${ }^{10,11}$ Masayuki Miyasaka, ${ }^{12,13}$ Kumar Sharma, ${ }^{14}$ Nagahiro Minato, ${ }^{2}$ Hiroshi Kawamoto, ${ }^{3}$ and Motoko Yanagita'

${ }^{1}$ Department of Nephrology, ${ }^{2}$ Department of Immunology and Cell Biology, ${ }^{5}$ Department of Urology, ${ }^{7}$ Medical Innovation Center, Graduate School of Medicine, ${ }^{3}$ Department of Immunology, Institute for Frontier Medical Science, Kyoto University, Kyoto, Japan. ${ }^{4}$ Department of Diagnostic Pathology, ${ }^{6}$ Department of Experimental Therapeutics, Institute for Advancement of Clinical and Translational Science, Kyoto University Hospital, Kyoto, Japan. ${ }^{8}$ Department of Immunology, Institute of Advanced Medicine, Wakayama Medical University, Wakayama, Japan. 'ªboratory for Metabolomics, RIKEN Center for Integrative Medical Sciences, Kanagawa, Japan. ${ }^{10}$ Department of Molecular Genetics, Howard Hughes Medical Institute, University of Texas Southwestern Medical Center, Dallas, Texas, USA. "International Institute for Integrative Sleep Medicine, University of Tsukuba, Tsukuba, Japan. ${ }^{12}$ Institute for Academic Initiatives, Osaka University, Osaka, Japan. ${ }^{13}$ MediCity Research Laboratory, University of Turku, Turku, Finland. ${ }^{14}$ Center for Renal Translational Medicine and Institute of Metabolomic Medicine, Department of Medicine, University of California San Diego, Veteran's Administration San Diego Health Care System, La Jolla, California, USA.

Acute kidney injury (AKI) is a common clinical condition defined as a rapid decline in kidney function. AKI is a global health burden, estimated to cause 2 million deaths annually worldwide. Unlike AKI in the young, which is reversible, AKI in the elderly often leads to end-stage renal disease, and the mechanism that prevents kidney repair in the elderly is unclear. Here we demonstrate that aged but not young mice developed multiple tertiary lymphoid tissues (TLTs) in the kidney after AKI. TLT size was associated with impaired renal function and increased expression of proinflammatory cytokines and homeostatic chemokines, indicating a possible contribution of TLTs to sustained inflammation after injury. Notably, resident fibroblasts from a single lineage diversified into 75 neurotrophin receptor $\left(p 75 \mathrm{NTR}^{+}\right)$fibroblasts and homeostatic chemokineproducing fibroblasts inside TLTs, and retinoic acid-producing fibroblasts around TLTs. Deletion of $\mathrm{CD}^{+}$cells as well as late administration of dexamethasone abolished TLTs and improved renal outcomes. Importantly, aged but not young human kidneys also formed TLTs that had cellular and molecular components similar to those of mouse TLTs. Therefore, the inhibition of TLT formation may offer a novel therapeutic strategy for AKI in the elderly. employed by the TMK Project after graduation. M. Yanagita is on the advisory board of Astellas and receives research grants from Astellas, Chugai, Daiichi Sankyo, Fujiyakuhin, Kyowa Hakko Kirin, Mitsubishi Tanabe Pharma Corporation, MSD, Nippon Boehringer Ingelheim, and Torii. S. Narumiya is supported by the Coordination Fund from JST and Astellas Pharma, Inc. S. Narumiya is a scientific advisor to Astellas Pharma.

Submitted: March 22, 2016

Accepted: June 13, 2016

Published: July 21, 2016

Reference information:

\section{Introduction}

Acute kidney injury (AKI) is a very common clinical condition characterized by a rapid fall in glomerular filtration rate, often as a result of an ischemic or nephrotoxic renal insult (1-3). AKI is associated with a high risk for mortality, subsequent chronic kidney disease (CKD), end-stage renal disease (ESRD), and other types of organ failure. The incidence of $\mathrm{AKI}$ is increasing worldwide, especially in the elderly population $(4,5)$, making AKI a major public health issue and an enormous global economic burden. In the United States alone, AKI affects more than 1.2 million patients per year during hospital stays, and kills 300,000 people annually; moreover, the population incidence rate of $\mathrm{AKI}$ is increasing an average of $10 \%$ per year $(1,4)$. While epidemiological studies showed that the clinical outcomes for AKI are worse in the elderly than in the young (5), the reasons for the failure of the aged kidney to repair itself and for its progression to CKD or ESRD after AKI have not been fully investigated. Thus, no effective therapeutic tools have been developed to improve survival after AKI in the elderly. 
Inflammation is a crucial contributor to cellular injury and malfunction $(2,3,6,7)$, and inflammatory cell infiltration is commonly observed in chronic diseases. Although most inflammatory cells are not spatially and functionally organized, chronic infiltrates sometimes organize into clusters that are enriched in $\mathrm{T}$ and $\mathrm{B}$ cells. These ectopic lymphoid structures in nonlymphoid organs are referred to as tertiary lymphoid tissues (TLTs) to distinguish them from secondary lymphoid organs (SLOs) such as lymph nodes (8-11). TLTs function as local sites of antigen presentation, clonal expansion, and lymphocyte activation, all of which discriminate TLTs from chronic infiltrates $(8,10,11)$. TLTs are supported structurally by nonhematopoietic stromal cells, fibroblasts in particular (8), although their function, developmental origin, and phenotypic heterogeneity remain unclear. TLTs often develop in a variety of chronic inflammatory lesions including autoimmunity, persistent infection, atherosclerosis, and cancer (8-11). Of particular interest, the role of TLTs is context dependent and can be beneficial or detrimental to the host. During infection, TLTs are often beneficial and are associated with pathogen clearance and increased survival (12). TLTs are detrimental when they destroy normal tissue and exacerbate autoimmune diseases and chronic rejection (8-11), suggesting that TLTs may serve as a therapeutic target in the latter contexts (13).

Here we demonstrated that aged mice, but not young mice, developed multiple renal TLTs in 3 kidney injury models, which promoted inflammation and might compromise kidney regeneration, and that the abolishment of TLTs improved renal outcomes. Notably, fibroblasts from a single lineage differentiated into several distinct phenotypes in response to their microenvironment, and these fibroblasts underlay TLT formation. Importantly, age-dependent TLT formation is also observed in human kidneys, and the cellular and molecular components of TLTs in humans are similar to those in mice. Thus, a mechanism similar to that observed in mice might contribute to the maladaptive repair of kidneys in elderly humans.

\section{Results}

Aged kidneys show multiple TLTs and sustained inflammation in response to injuries. Before the induction of AKI, no difference was observed in the renal function or morphology between young (8-week-old) and aged (12-month-old) mice (Supplemental Figure 1; supplemental material available online with this article; doi:10.1172/jci.insight.87680DS1). To evaluate the impact of aging on the prognosis of AKI, we induced unilateral ischemic reperfusion injury (IRI), and found that aged kidneys showed interstitial fibrosis, delayed regeneration, and persistent inflammation (Figure 1, A and B, and Supplemental Figure 2, A and B). Notably, aged but not young kidneys exhibited significant inflammatory cell aggregates after IRI (Figure 1A); these aggregates were distributed in the cortex and along the hilus of the kidney (Supplemental Figure 2C), and also contained arteries, lymphatic vessels, and high endothelial venules (HEVs) (Figure 1, C and D). T cells and B cells were predominant and intermingled throughout the aggregates (Figure 1E). The $\mathrm{T}$ cells consisted mainly of $\mathrm{CD}^{+} \mathrm{T}$ cells (Supplemental Figure 3, A and B). Some dendritic cells were also identified as the third population (Supplemental Figure 3, C and D). These aggregates contained a large number of proliferative $\left(\mathrm{Ki}^{+} 7^{+}\right.$) cells (Figure 1F), some of which were also positive for CD3 $\varepsilon$ and B220 (Supplemental Figure 3, E-G). Furthermore, we also detected T cells positive for programmed death-1 (PD-1, CD279), a negative checkpoint regulator expressed on antigen-stimulated $\mathrm{T}$ cells $(14,15)$, within these aggregates (Supplemental Figure 3H). These results suggest that the T-B cell interactions occurred within these lymphoid aggregates, which therefore allows us to define these lymphoid aggregates as TLTs.

As ischemic time or the time after IRI increased, TLTs expanded, appeared to destroy adjacent healthy nephrons, and occupied larger areas of the renal parenchyma in aged kidneys (Figure 1, G and I, and Supplemental Figure 3I). In contrast, TLT formation was almost undetectable in young kidneys even after 60-minute IRI, which causes very severe injury (Figure 1, H and I, and Supplemental Figure 3J), suggesting that aging itself is a predisposing factor for TLT formation after kidney injury. Moreover, the expression of Ifng and Tnfa was upregulated in aged kidneys (Figure 1J) and was closely associated with TLT sizes (Figure 1K). In 2 other models of kidney injury, the folic acid (FA) nephropathy model and the unilateral ureteral obstruction (UUO) model, TLTs were again found in aged kidneys, but were almost undetectable in young kidneys (Figure 1L and Supplemental Figure 4). In the FA nephropathy model, the degree of impairment of renal functions was worse in aged mice and correlated well with TLT size (Figure 1M). Thus, TLTs form in aged kidneys after injury, regardless of the type of injury, and could serve as a marker of sustained injury and inflammation.

Fibroblasts within TLTs produce homeostatic chemokines. TLT formation mimics, at least in part, the developmental program of SLOs $(8,10,11)$. Accordingly, we analyzed the renal expression of the homeostatic 
A Young 30-min IRI
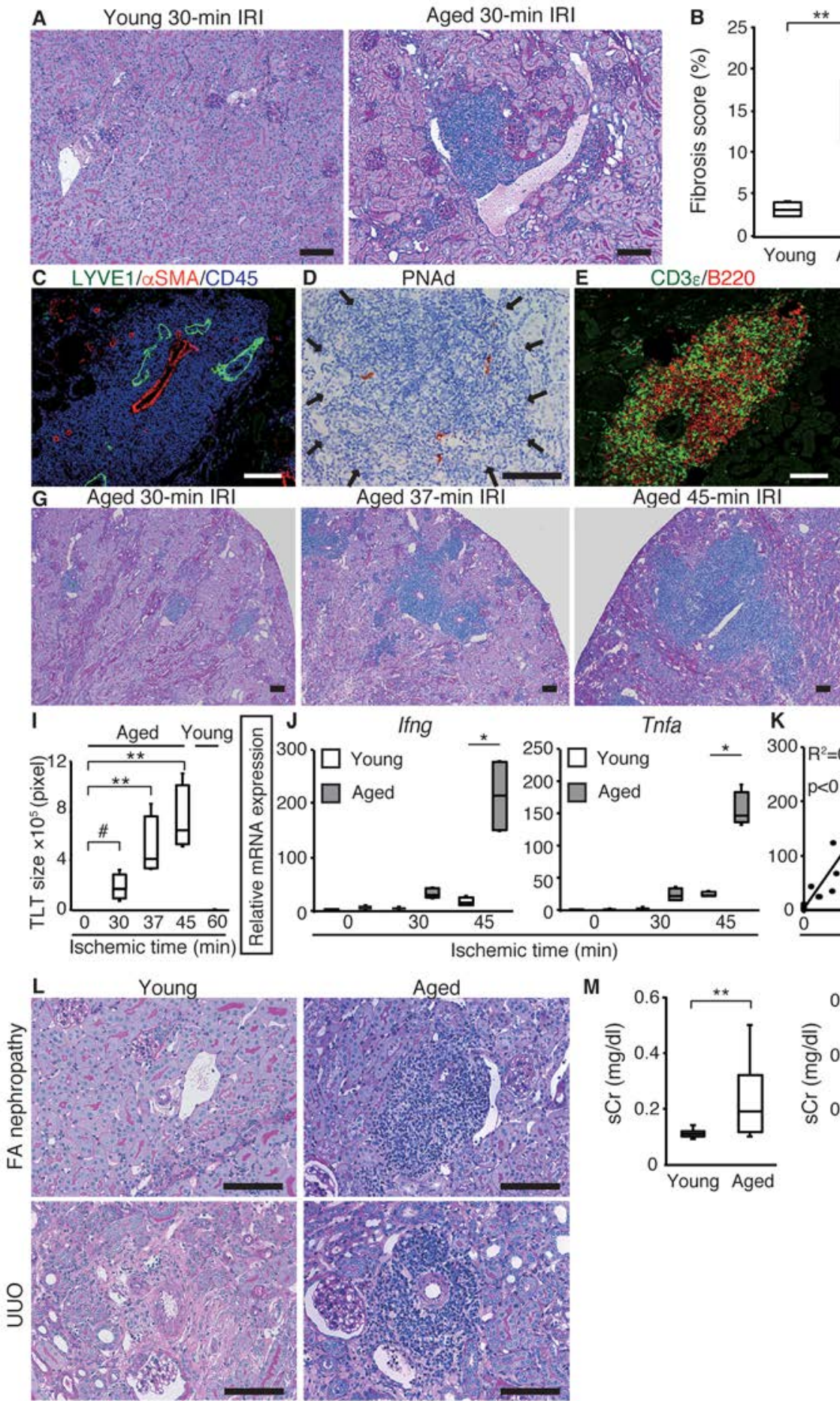

Aged 45-min IRI
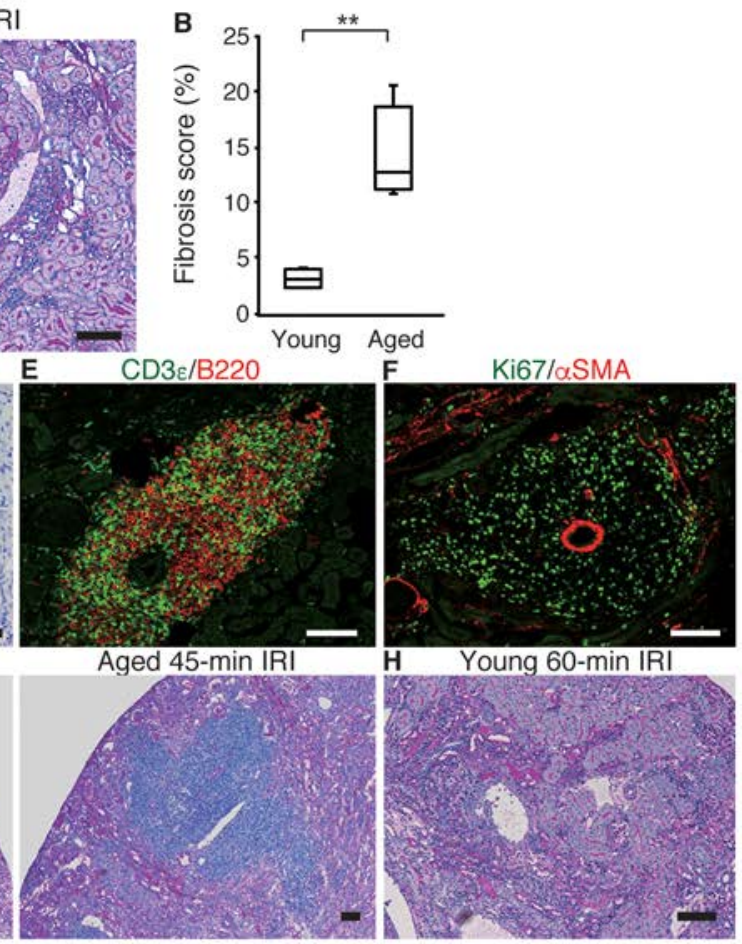

H Young 60 -min IRI

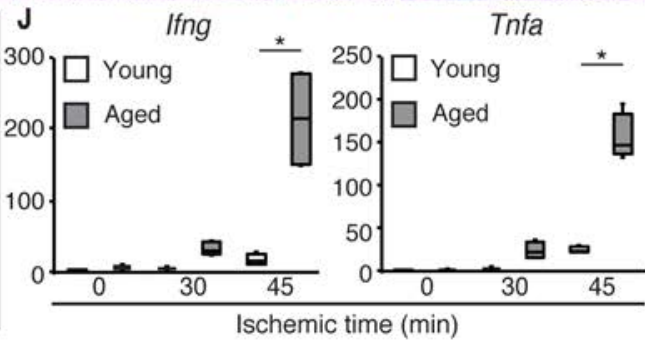

$\mathbf{K}$

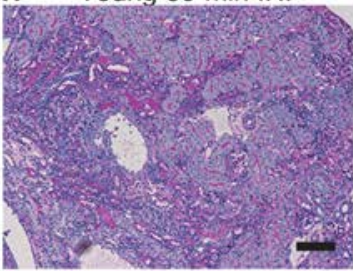

Ifng Tnfa

${ }^{300} R^{2}=0.724 . \quad{ }^{250} R^{2}=0.813$
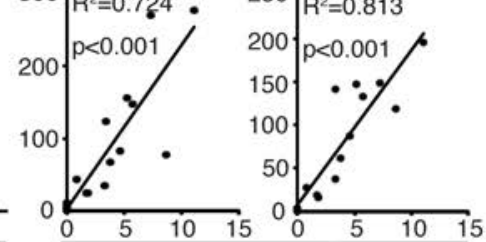

TLT size $\times 10^{5}$ (pixel)

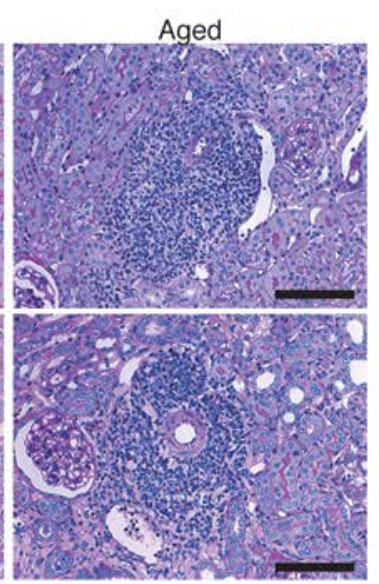

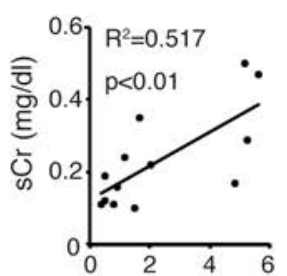

TLT size $\times 10^{5}$ (pixel)

Figure 1. Aged mice develop multiple renal tertiary lymphoid tissues (TLTs) after kidney injury. (A) Periodic acid-Schiff (PAS) staining and (B) fibrosis scores 45 days after 30-minute ischemic reperfusion injury (IRI) in young and aged mice ( $n=4$ per group). (C, E, and F) Immunofluorescence analysis of (C) lymphatic vessel endothelial hyaluronan receptor 1 (LYVE1), $\alpha$-smooth muscle actin ( $\alpha$ SMA), and CD45; (E) CD3e and B220; (F) Ki67 and $\alpha$ SMA; and (D) immunohistochemical analysis of peripheral lymph node addressin (PNAd) in inflammatory cell aggregates in aged kidneys at day 45 after $37-m i n u t e ~ I R I$. (G and $\mathbf{H}$ ) PAS staining of IRI kidneys of (G) aged mice and (H) young mice at day 45 , and (I) the TLT sizes ( $n=4$ or 5 per group). (J) Ifng and Tnfa mRNA levels of IRI kidneys at day 45 with variable ischemic times in young and aged mice ( $n=4$ per group). (K) Correlation between TLT sizes and mRNA levels of Ifng and Tnfa in aged IRI kidneys at day 45 with variable ischemic times $(n=16)$. (L) PAS staining of young and aged kidneys in folic acid (FA) nephropathy (day 21) and unilateral ureteral obstruction (UUO) (day 14) model. (M) Serum creatinine (sCr) concentrations in young and aged mice 21 days after FA treatment ( $n=12$ or 13 per group) and correlation of sCr with TLT size in aged mice $(n=13) .{ }^{*} P<0.001,{ }^{* *} P<0.01,{ }^{\#} P<0.05$ versus control. One-way ANOVA with Tukey's post-hoc analysis was used to analyze data from (J); a 2-tailed Student's $t$ test was used for other experiments. Correlation was determined by Pearson's correlation analysis. Scale bars: (A, C-H, and $\mathbf{L}) 100 \mu \mathrm{m}$. The expression levels were normalized to those of Gapdh and expressed relative to those of young mouse kidney at day $\mathbf{0}(\mathrm{IRI})$. In (B, I, J, and $\mathbf{M}$ ), the box corresponds to the first quartile, median (horizontal bar in the box), and third quartile, and the whiskers extend from minimum to maximum values. 


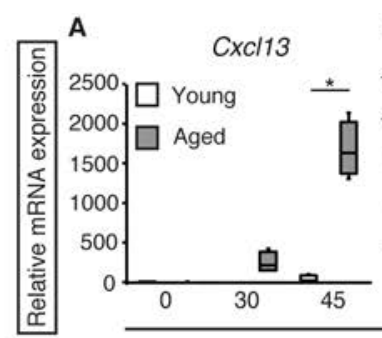

B

C

Ccl21
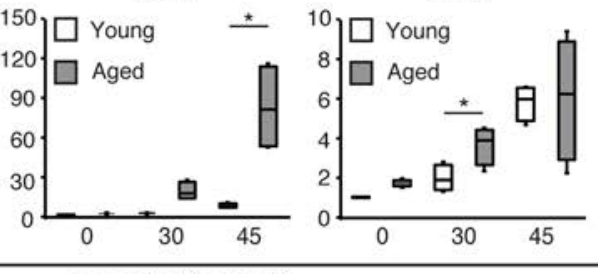

schemic time ( $\mathrm{min})$
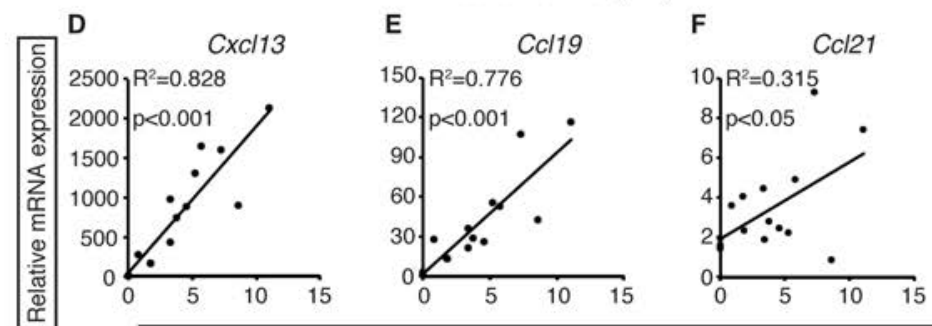

TLT size $\times 10^{5}$ (pixel)
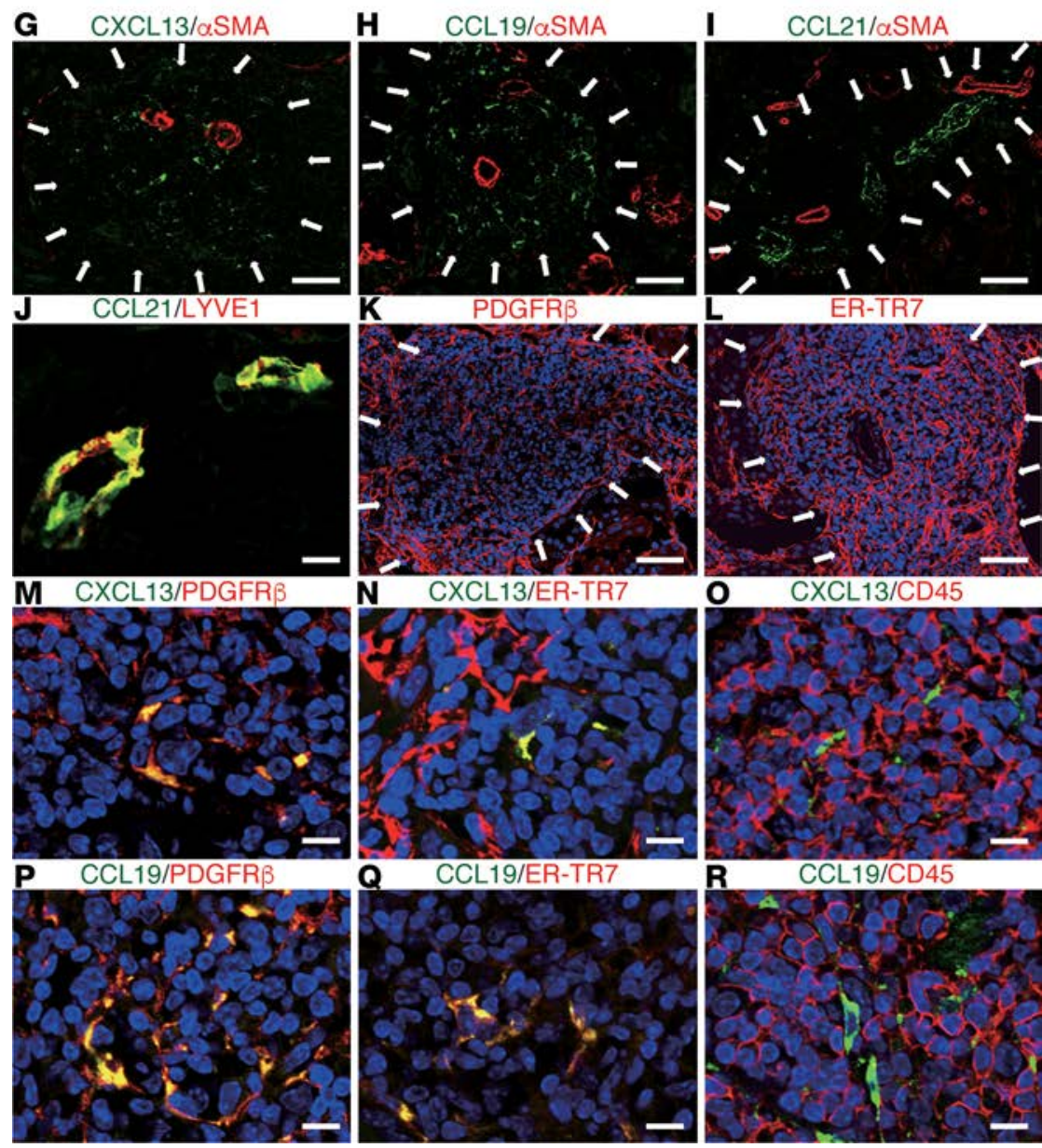

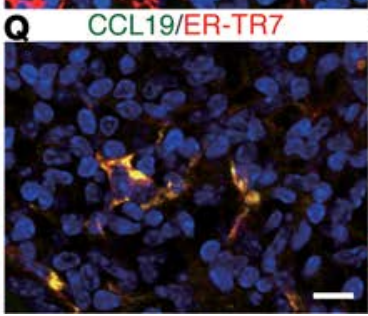

\section{CCL19/ER-TR7}
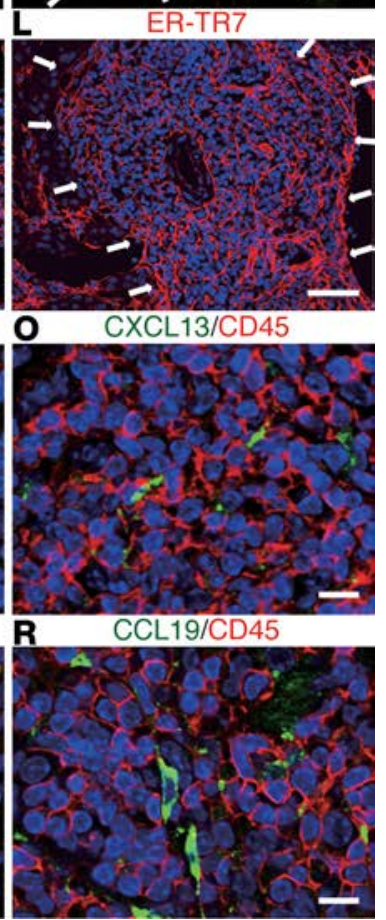

Figure 2. Fibroblasts inside tertiary lymphoid tissues (TLTs) produce CXCL13 and CCL19. (A-C) The mRNA expression of the homeostatic chemokines Cxcl13, Cc/19, and Cc/21 in ischemic reperfusion injury (IRI) kidneys 45 days after variable ischemic time IRI in young and aged mice ( $n=4$ per group). The expression levels were normalized to those of Gapdh and expressed relative to those of young mouse kidney at day 0 (IRI). ${ }^{*} P<0.001,{ }^{* *} P<0.01$ aged versus young (1-way ANOVA with Tukey's post-hoc analysis). The box corresponds to the first quartile, median (horizontal bar in the box), and third quartile, and the whiskers extend from minimum to maximum values. (D-F) Correlations between TLT sizes and mRNA levels of $C x c / 13, C c / 19$, and $C c / 21$ in aged IRI kidneys 45 days after variable ischemic time IRI $(n=16)$. Correlation was determined by Pearson's correlation analysis. (G-R) Immunohistological analysis of aged kidneys 30 days after 37-minute IRI. Immunofluorescence analysis of (G-I) CXCL13, CCL19, and CCL21 with $\alpha$-smooth muscle actin ( $\alpha S M A) ;(J)$ CCL21 and lymphatic vessel endothelial hyaluronan receptor 1 (LYVE1); (K) PDGFR $\beta$; (L) ER-TR7; (M-0) PDGFR $\beta$, ER-TR7, and CD45 with CXCL13; and (P-R) PDGFR $\beta$, ER-TR7, and CD45 with CCL19. Arrows indicate TLT localization. Scale bars: (G-I) $100 \mu \mathrm{m}$, (K and L) $50 \mu \mathrm{m},(\mathbf{J}, \mathbf{M}-\mathbf{R}) 10 \mu \mathrm{m}$.

chemokines CXCL13, CCL19, and CCL21, all of which are essential for SLO development. Renal Cxcl13 and Ccl19 expression increased dramatically in an ischemic time-dependent manner in aged kidneys, but not in young kidneys (Figure 2, A and B). Ccl21 expression was increased to a lesser extent, although the expression was comparable between both groups (Figure 2C). In addition, Cxcl13 and Ccl19 expression correlated strongly, whereas Ccl21 expression correlated weakly, with the sizes of TLTs (Figure 2, D-F). Renal Cxcl13 and Ccl19 expression also increased during the course of TLT formation (Supplemental Figure 5).

CXCL13 and CCL19 signals were confined within the TLTs (Figure 2, G and H), whereas CCL21 signals were detected in lymphatic vessels (Figure 2, I and J). As with SLOs, the renal TLTs contained a fibroblast reticular network positive for both PDGFR $\beta$ and the fibroblast marker ER-TR7 (Figure 2, K and L). Notably, the CXCL13- and CCL19-positive cells within the TLTs were also positive for PDGFR $\beta$ and

ER-TR7, but not for CD45 or CD11c (Figure 2, M-R, and Supplemental Figure 6). These results suggest that renal fibroblasts are the main source of CXCL13 and CCL19 in aged kidneys.

Fibroblasts with distinct phenotypes underlie TLT formation in aged injured kidneys. Crosstalk between lymphoid tissue organizer cells and lymphoid tissue inducer cells plays a central role in SLO development (10), and this crosstalk is triggered by retinoic acid (RA) released from the neurons $(10,16)$. While neural fibers in the kidney were negative for retinaldehyde dehydrogenase 2 (RALDH2) (Figure 3B), which is the rate-limiting enzyme in RA synthesis (17), fibroblasts outside the TLTs were strongly positive for RALDH2 in the early 
A

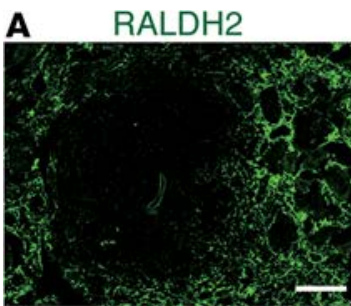

B 33 -tubulin/RALDH2/DAPI

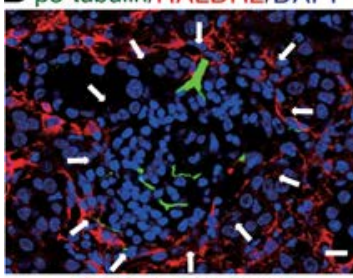

F $\mathrm{CD} 21 / \mathrm{CXCL} 13$

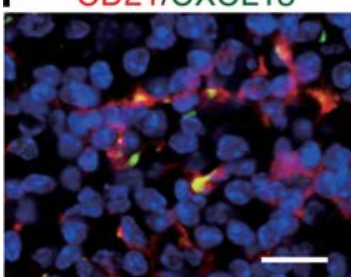

H

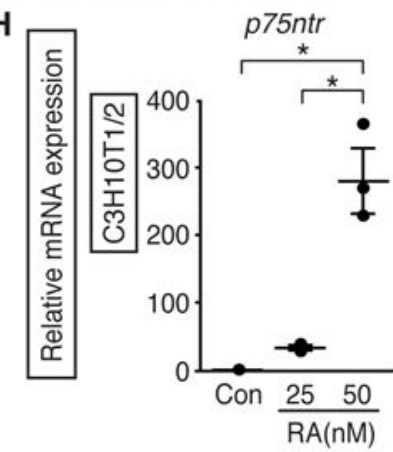

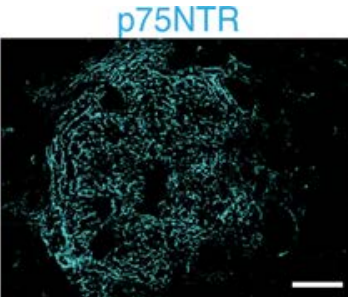
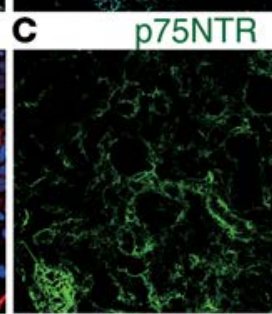

G
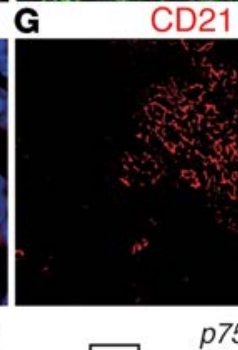
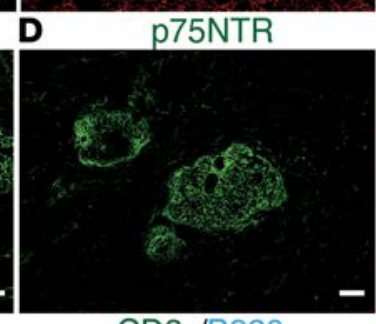

$\mathrm{CD} 3 \varepsilon / \mathrm{B} 220$
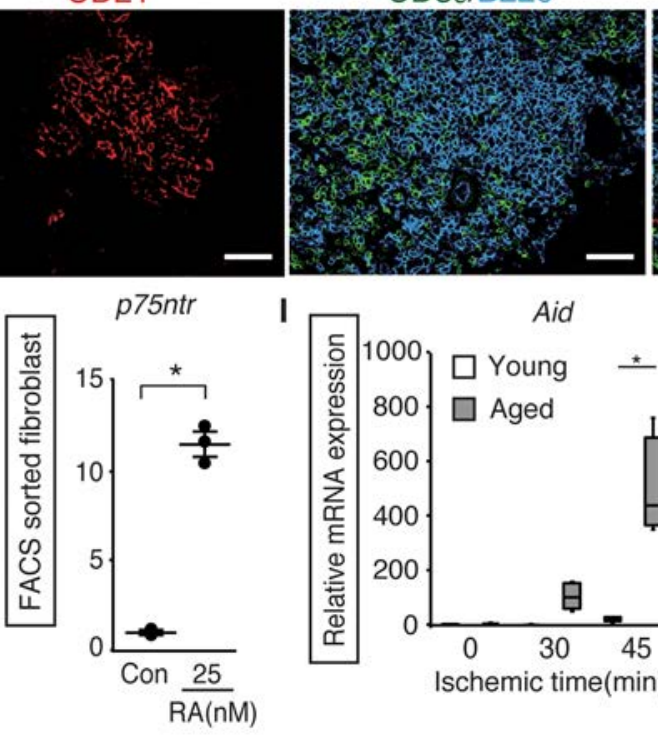

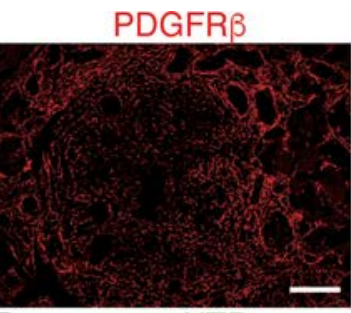

RALDH2/PDGFR $3 / p 75 N T R$
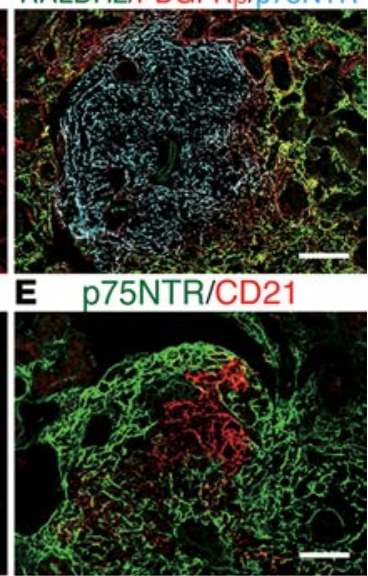

CD3ع/CD21/B220

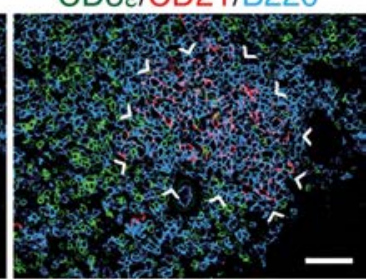

Aid

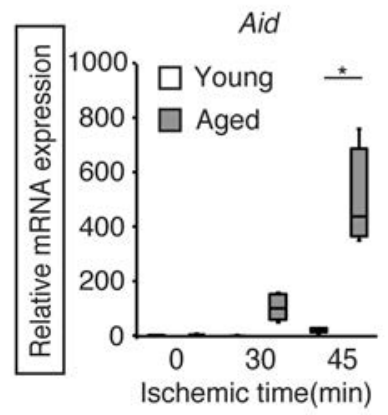

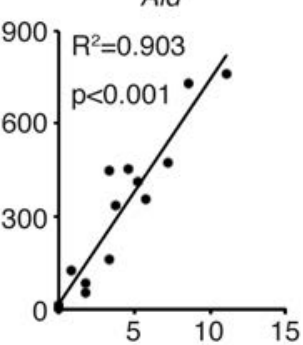

TLT size $\times 10^{5}$ (pixel)

Figure 3. Fibroblasts with distinct phenotypes underlie tertiary lymphoid tissue (TLT) formation in the aged injured kidney. Immunofluorescence of (A) retinaldehyde dehydrogenase 2 (RALDH2), p75 neurotrophin receptor (p75NTR), and PDGFR $\beta$; (B) $\beta 3$-tubulin and RALDH2; (C and D) p75NTR; (E) p75NTR and CD21; (F) CD21 and CXCL13; and (G) CD3e, CD21, and B220 in aged kidneys after ischemic reperfusion injury (IRI). Arrows indicate TLT localization. Arrowheads indicate the localization of the B cell area. Aged kidneys were analyzed 14 (B and C), 30 (A), and 45 (D-C) days after IRI. (H) Retinoic acid (RA) induces $p 75 n t r$ mRNA expression in C3H10T1/2 mouse embryonic fibroblasts and FACS-sorted PDCFR $\beta^{+}$ cells ( $n=3$ per group). The data are presented as dot plots (mean \pm SD). (I) Activation-induced cytidine deaminase (Aid) mRNA levels of kidneys 45 days after various ischemic time IRI in young and aged mice ( $n=4$ per group) and correlation with TLT size ( $n=16$, aged mice only). The expression levels were normalized to those of Gapdh and expressed relative to those of controls or young mouse kidney at day 0 (IRI). ${ }^{*} P<0.001$ versus controls. A 2-tailed Student's $t$ test was used to analyze data from FACS-sorted PDGFR $\beta^{+}$cells; 1-way ANOVA with Tukey's post-hoc analysis was used for other experiments. Correlation was determined by Pearson's correlation analysis. The box corresponds to the first quartile, median (horizontal bar in the box), and third quartile, and the whiskers extend from minimum to maximum values. Scale bars: (A, C, and D) $100 \mu \mathrm{m}$, (E and G) $50 \mu \mathrm{m}$, (B and F) $10 \mu \mathrm{m}$.

phase of TLT formation (Figure 3A). Interestingly, the fibroblasts inside the TLTs did not express RALDH2, but they did express p75 neurotrophin receptor (p75NTR) (Figure 3A), a marker for the neural crest (18). In the early phase of IRI, p75NTR signals were detectable in the broad area of the renal interstitium (Figure 3C), whereas in the later phase, the signals gradually disappeared in the interstitium and became confined to the fibroblasts inside the TLTs (Figure 3D). We previously demonstrated that p75NTR is expressed in fibroblasts in neonatal kidneys, but disappears in healthy adult kidneys (19). Fibroblasts inside the TLTs seemed to retrieve the phenotype of the fibroblasts in the neonatal kidneys. RALDH2 $2^{+}$fibroblasts surrounded p75NTR ${ }^{+}$ fibroblasts in TLTs; moreover, RA treatment significantly upregulated the $p 75 \mathrm{ntr}$ expression in C3H10T1/2 mouse embryonic fibroblasts and FACS-sorted PDGFR $\beta^{+}$mouse kidney fibroblasts (Figure $3 \mathrm{H}$ ). 

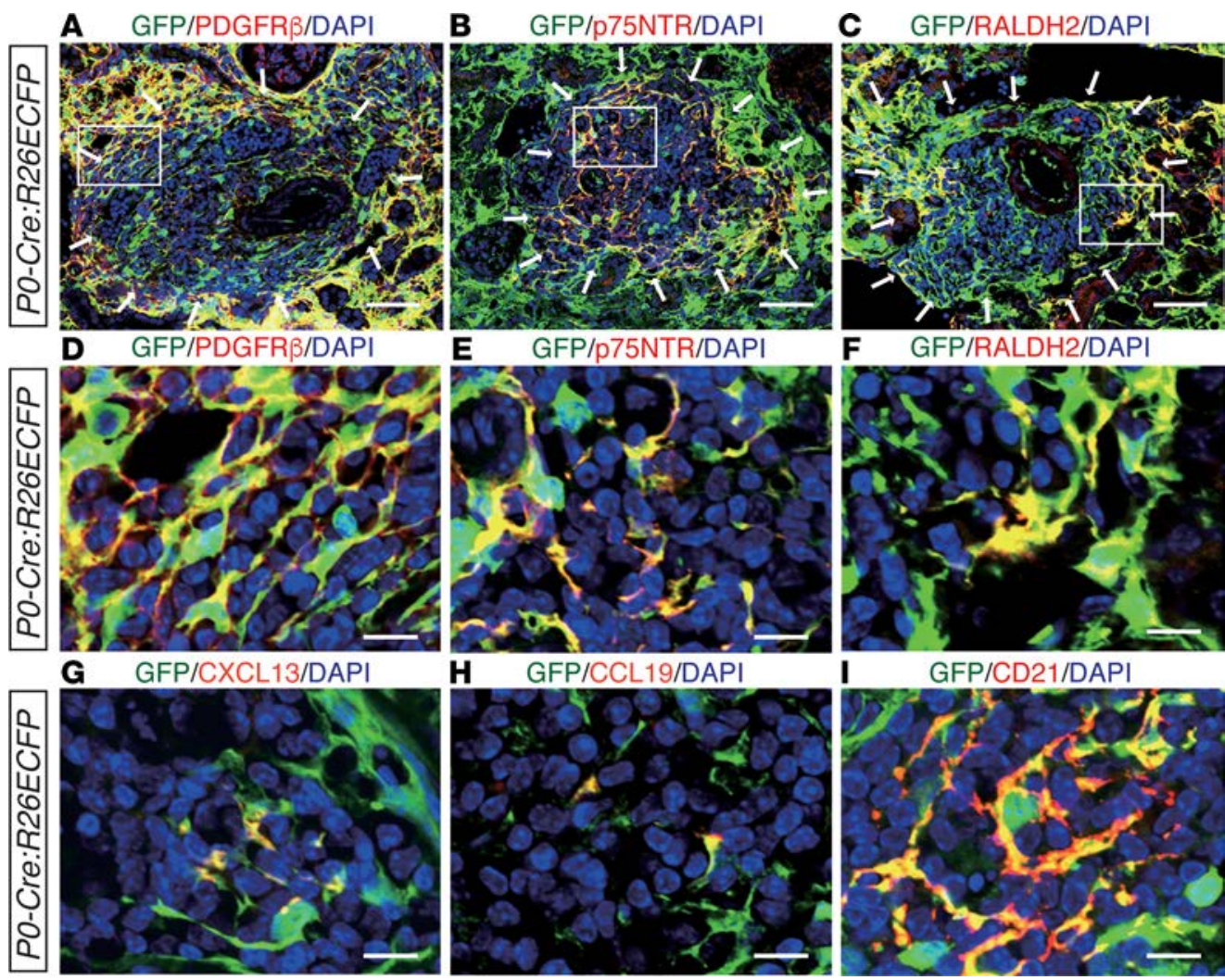

Figure 4. Myelin protein zero-Cre ( $P O-C r e)$ lineage-labeled fibroblasts differentiate into diverse fibroblasts with distinct phenotypes. (A-I) Immunofluorescence (IF) of enhanced cyan fluorescent protein-positive (ECFP+) cells in kidneys subjected to ischemic reperfusion injury (IRI) in aged PO-Cre/R26ECFP mice. ECFP was visualized with an anti-GFP antibody. IF of GFP and (A and $\mathbf{D})$ PDGFR $\beta$, (B and E) p75 neurotrophin receptor (p75NTR), (C and $\mathbf{F}$ ) retinaldehyde dehydrogenase 2 (RALDH2), (G) CXCL13, (H) CCL19, and (I) CD21. Magnified views of the outlined regions in (A-C) are shown in (D-F), respectively. Arrows in (A-C) indicate tertiary lymphoid tissue (TLT) localization. Scale bars: (A-C) $50 \mu \mathrm{m},(\mathbf{D}-\mathbf{I}) 10 \mu \mathrm{m}$.

In the later phase of the injury, p75NTR-negative areas began to appear within the TLTs, and CD21 ${ }^{+}$ stromal cells emerged and compensated for the p75NTR-negative areas (Figure 3E). CD21+ stromal cells expressed CXCL13 (Figure 3F) and formed B cell areas (Figure 3G), indicating the development of follicular dendritic cells (FDCs), which are conventional CXCL13-producing stromal cells found in SLOs (20), as a part of the stromal network. FDCs are known to support germinal center responses in SLOs, and the expression of activation-induced cytidine deaminase ( $\mathrm{Aid}$ ), which is essential for both somatic hypermutation and class switch recombination in germinal center B cells (21), increased in an ischemic time-dependent manner and correlated closely with TLT size in aged kidneys (Figure 3I). These results suggest that a germinal center response may occur during the later phase of TLT formation.

Heterogeneous fibroblasts involved in TLT formation are derived from a single lineage. Although the phenotypic diversity of fibroblasts associated with TLTs was established above, the origin of these heterogeneous fibroblasts was still unknown. We previously showed that renal resident fibroblasts in the cortex and outer medulla are lineagelabeled with myelin protein zero-Cre ( $\mathrm{PO}-\mathrm{Cre}$; a Cre strain that expresses $\mathrm{Cre}$ in the migrating neural crest and Schwann cells; see ref. 22) and that P0-Cre lineage-labeled resident fibroblasts transdifferentiate into $\alpha \mathrm{SMA}^{+}$ myofibroblasts and drive renal fibrosis in the injured kidney $(19,23)$. To examine the contribution of $P 0$-Cre lineage-labeled resident fibroblasts to the heterogeneous fibroblasts in TLTs, we used P0-Cre:R26ECFP mice to trace the lineage of these fibroblasts. We found that almost all PDGFR $\beta^{+}$fibroblasts both inside and outside of TLTs, as well as p75NTR ${ }^{+}, \mathrm{RALDH} 2^{+}, \mathrm{CXCL}_{13}{ }^{+}, \mathrm{CCL} 19^{+}$, and $\mathrm{CD} 21^{+}$fibroblasts, were lineage-labeled with P0-Cre (Figure 4, A-I). These data support the idea that P0-Cre lineage-labeled fibroblasts diversify into heterogeneous fibroblasts with several different phenotypes in response to their microenvironment, and also underlie TLT formation. Neither Cre protein nor P0 and Cre mRNA was detected in aged P0-Cre:R26ECFP kidneys (Supplemental Figure 7), thereby excluding the possibility of Cre reactivation in the kidneys with TLTs. 

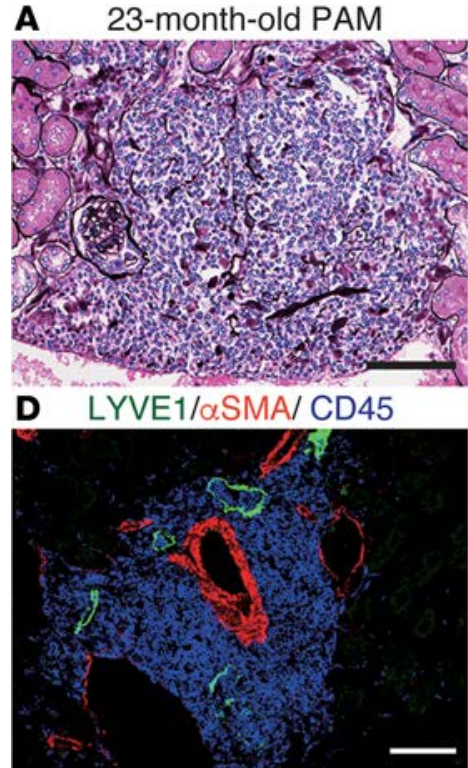

G

p75NTR
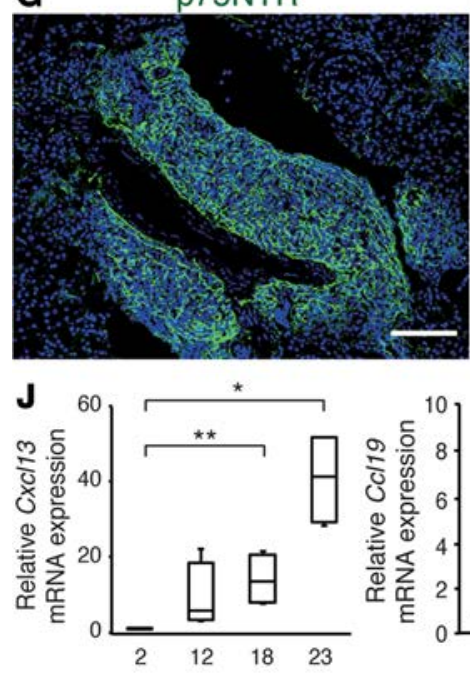

B

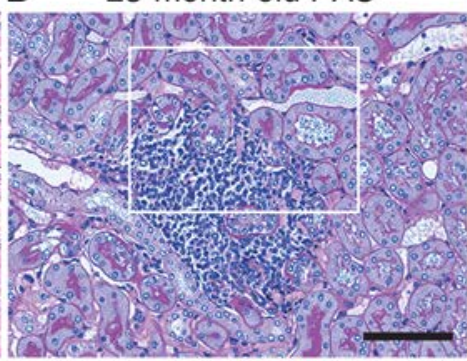

E

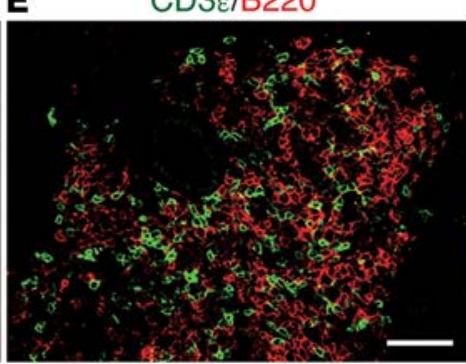

H CXCL13/aSMA

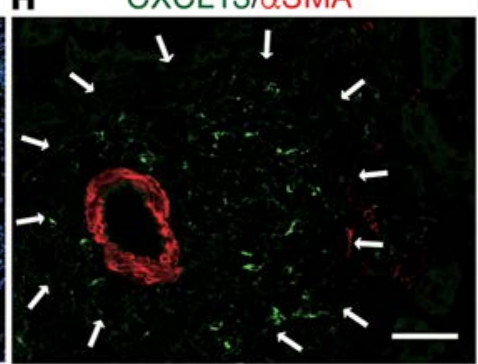

$\star \star$

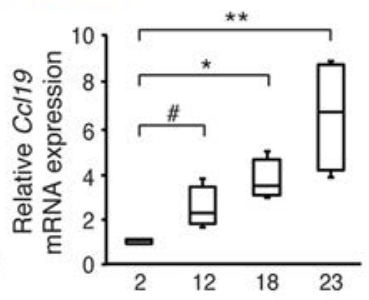

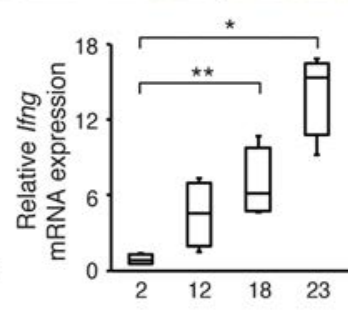

$\begin{array}{ll}\mathbf{K} & \mathrm{Ki} 67\end{array}$

C 23-month-old PAS
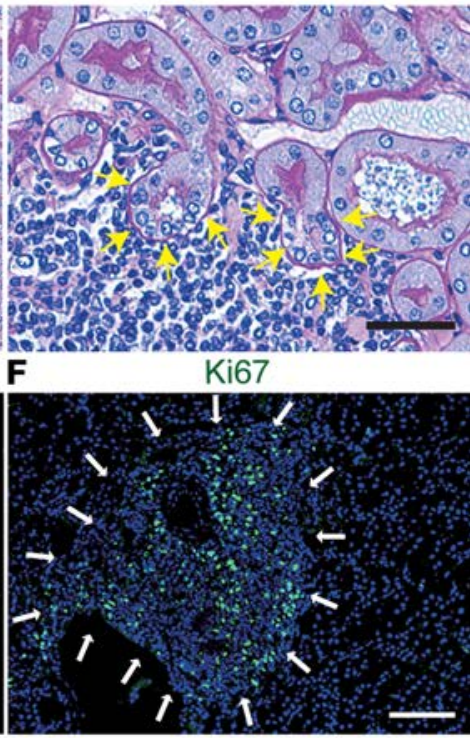

I CCL19/aSMA

Age (months)

Figure 5. Superaged mice spontaneously develop renal tertiary lymphoid tissues (TLTs) without injury. (A-I) Histological analysis of a 23-month-old mouse kidney. (A) Periodic acid-methenamine silver (PAM) staining showing a TLT containing PAM-positive basement membranes of nephron segments. (B and C) PAS staining showed signs of injury (yellow arrows) in tubules adjacent to TLTs. A magnified view of the outlined region in $\mathbf{B}$ is shown in C. (D-I) Immunofluorescence analysis of (D) Iymphatic vessel endothelial hyaluronan receptor 1 (LYVE1), $\alpha$-smooth muscle actin ( $\alpha$ SMA), and CD45; (E) CD3 $\varepsilon$ and B220; (F) Ki67; (C) p75 neurotrophin receptor (p75NTR); (H) CXCL13 and $\alpha$ SMA; and (I) CCL19 and $\alpha$ SMA. White arrows indicate the localization of TLTs. Scale bars: (A, B, D, F, G, and I) $100 \mu \mathrm{m}$, (C, E, and H) $50 \mu \mathrm{m}$. (J) The expression of Cxc/13, Cc/19, Ifng, and Tnfa mRNA in mouse kidneys at various ages ( $n=4$ per group). The expression levels were normalized to those of Gapdh and expressed relative to those of young (2-month-old) mouse kidneys. In J, the box corresponds to the first quartile, median (horizontal bar in the box), and third quartile, and the whiskers extend from minimum to maximum values.

${ }^{*} P<0.001,{ }^{* *} P<0.01,{ }^{*} P<0.05$ versus values at 2 months of age ( 2 -tailed Student's $t$ test).

Superaged mice spontaneously develop TLTs without injury. Aging is often accompanied by chronic inflammation that leads to a functional decline in various organs (24). Even without kidney injury, superaged mice developed renal TLT-like structures (Figure 5, A-C), which entangled the glomeruli and tubules inside (Figure 5A) and appeared to damage adjacent tubules (Figure 5, B and C). As with the TLTs in aged injured kidneys, these structures surrounded the interlobular artery and lymphatic vessel endothelial hyaluronan receptor $1^{+}\left(\mathrm{LYVE} 1^{+}\right)$lymphatic vessels (Figure 5D). These TLT-like structures were composed mainly of $\mathrm{T}$ cells and $\mathrm{B}$ cells (Figure $5 \mathrm{E}$ ), some of which were $\mathrm{Ki67}^{+}$(Figure $5 \mathrm{~F}$ ), thereby defining these 
A

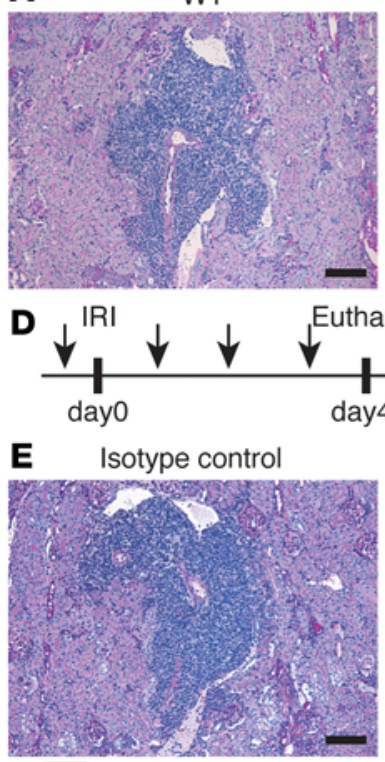

CXCL13 KO

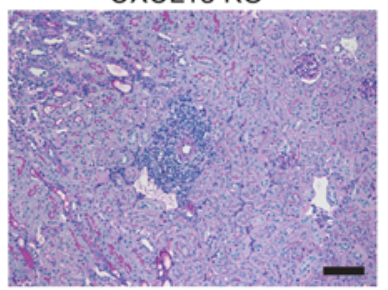

: GK1.5 or Isotype antibody
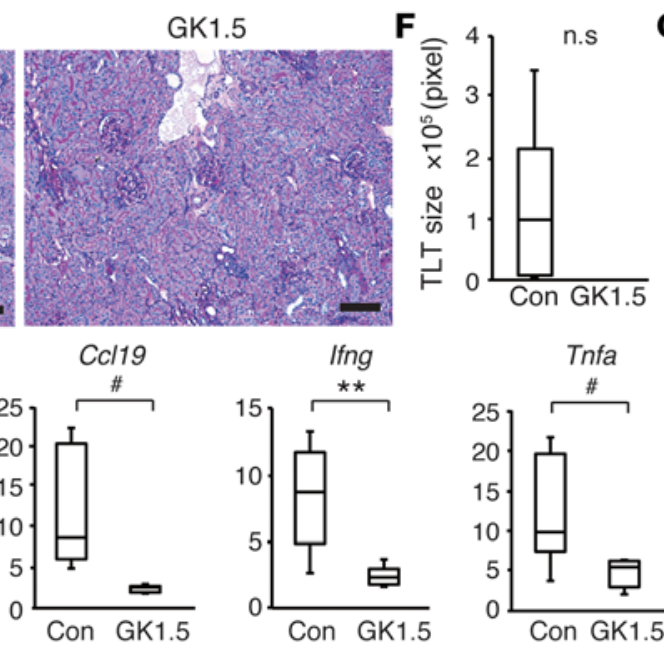

B

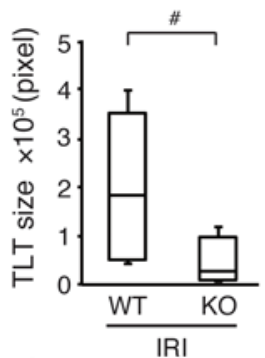

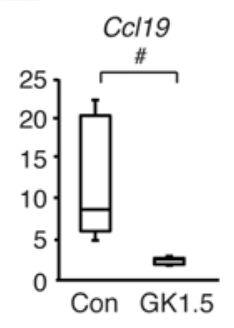

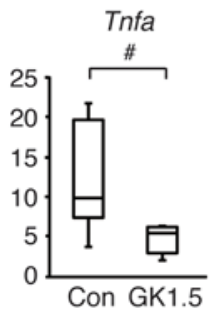

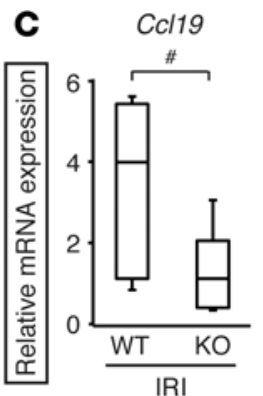
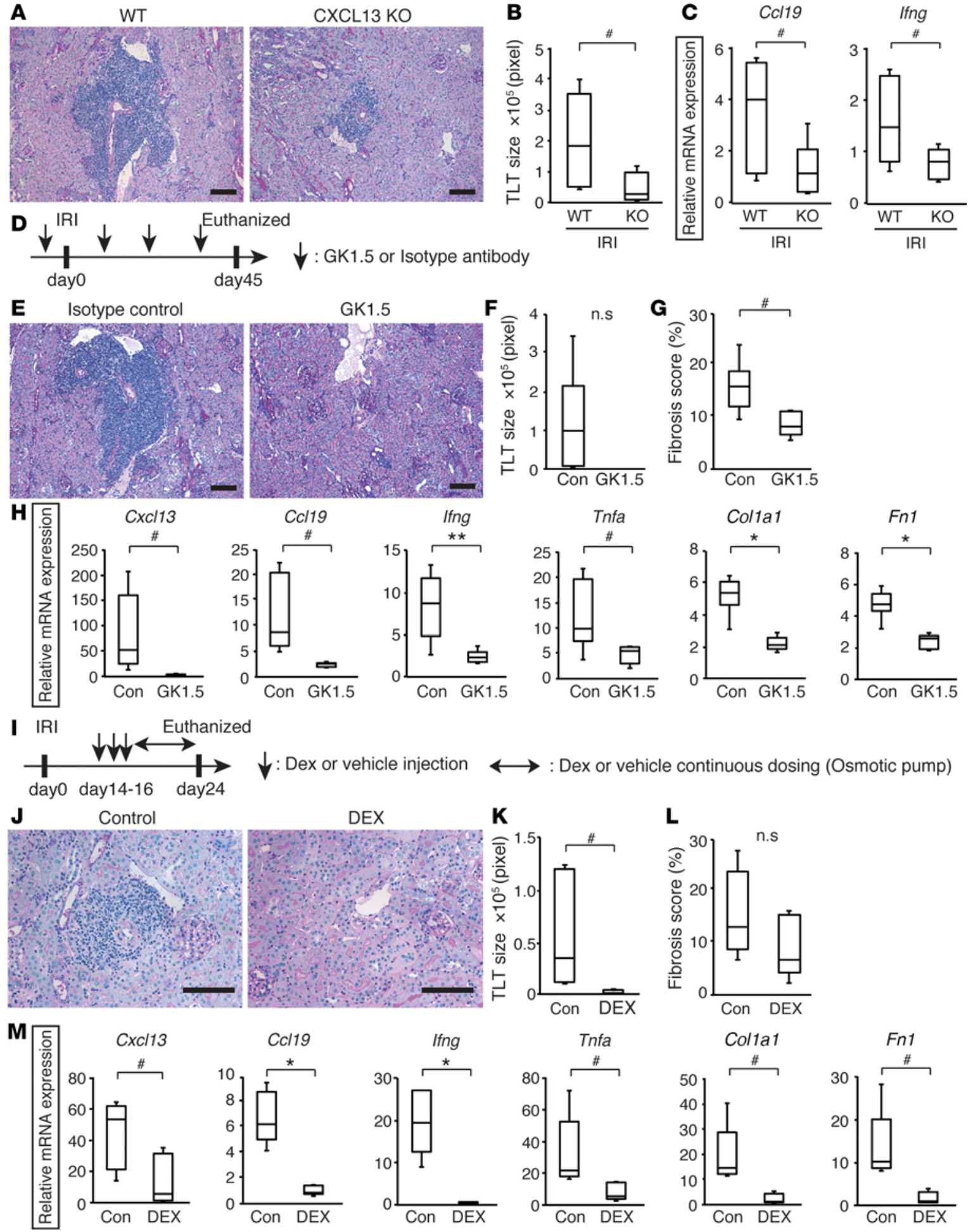

Figure 6. Targeting tertiary lymphoid tissue (TLT) formation has the potential to ameliorate renal fibrosis and inflammation. (A-C) Analysis of the kidneys from aged CXCL13-deficient mice (KO) and their littermates (WT) 45 days after ischemic reperfusion injury (IRI) ( $n=6$ per group). (A) Periodic acidSchiff (PAS) staining, (B) TLT sizes, and (C) Ccl19 and Ifng mRNA levels in IRI kidneys. (D-H) GK1.5 treatment study ( $n=5$ or 6 per group). (D) Experimental protocol, (E) PAS staining, (F) TLT sizes, (C) fibrosis scores, (H) Cxcl13, Ccl19, Ifng, Tnfa, Col1a1 (type 1 collagen $\alpha-1$ subunit), and Fn1 (fibronectin) mRNA levels in the IRI kidneys of GK1.5-treated mice (GK1.5) and isotype antibody-treated mice (Isotype control, Con). (I-M) Dexamethasone (Dex) treatment study ( $n=5$ per group). (I) Experimental protocol, (J) PAS staining, (K) TLT sizes, (L) fibrosis scores, and (M) Cxcl13, Ccl19, Ifng, Tnfa, Col1a1, and Fn1 mRNA levels in the IRI kidneys of Dex-treated (DEX) and vehicle-treated mice (Control, Con). Mice were subjected to 37-minute IRI (A-C) or 30-minute IRI (D-M). The expression levels were normalized to those of Gapdh and expressed relative to those of aged (12-month-old) mouse kidney. The box corresponds to the first quartile, median (horizontal bar in the box), and third quartile, and the whiskers extend from minimum to maximum values. ${ }^{*} P<0.001,{ }^{* *} P<0.01$, $\# P<0.05$ versus control (2-tailed Student's $t$ test). n.s, not significant. Scale bars: (A, E, and J) $100 \mu \mathrm{m}$. 
A
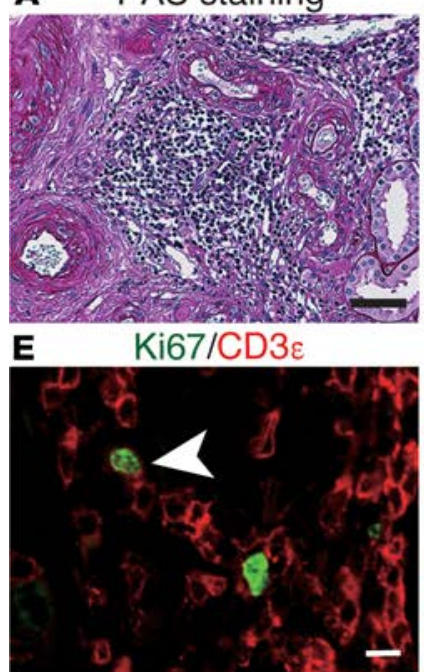

H
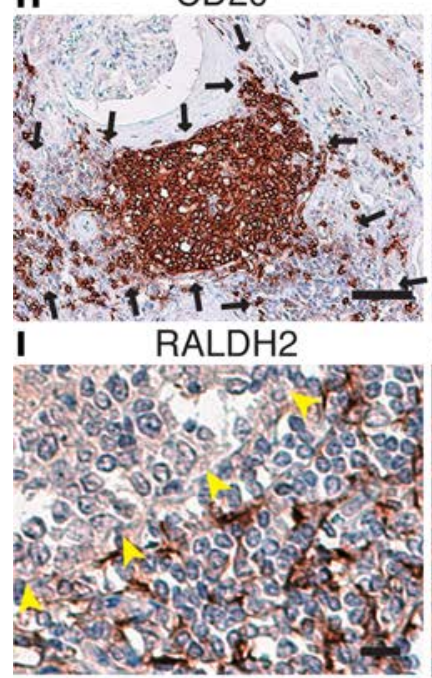

M

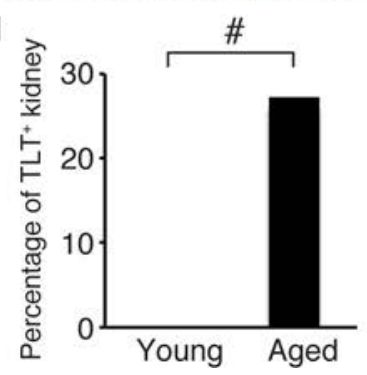

B PAS staining

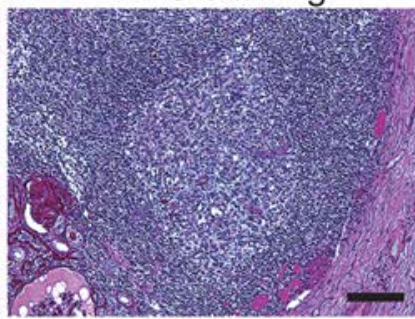

Ki67/CD20

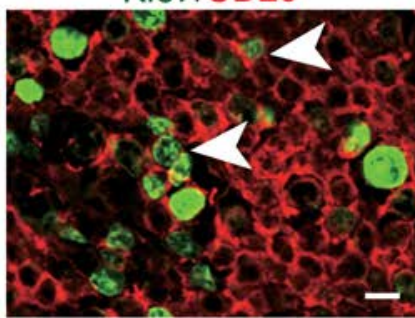

AID

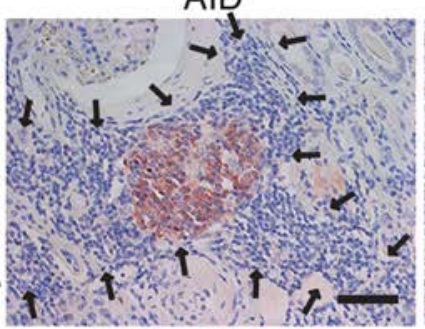

J $\quad$ CXCL13/CD45

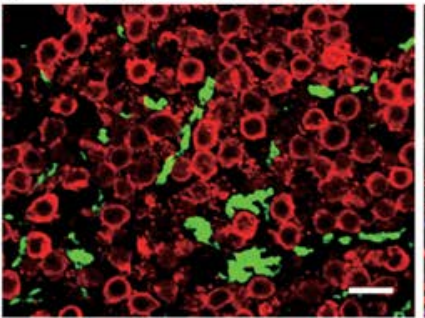

c $\quad \mathrm{CD} 20 / \mathrm{CD} 3 \varepsilon$

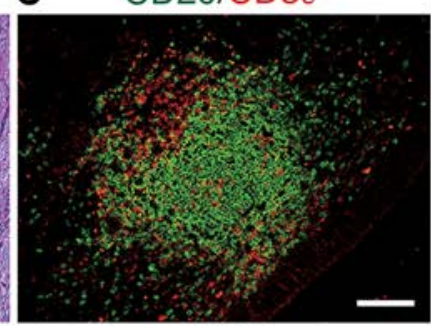

F

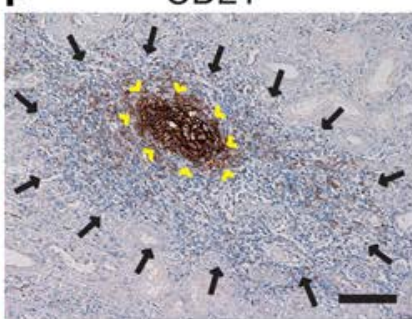

CD21

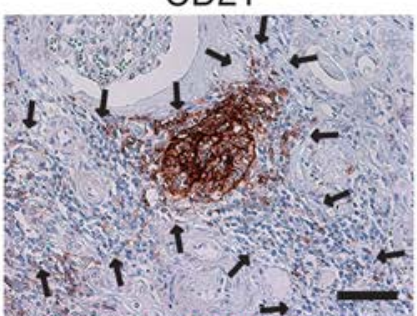

K CD21/aSMA/CD 45

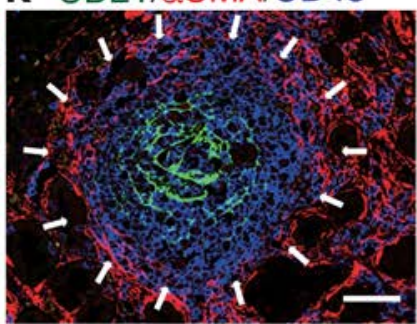

D

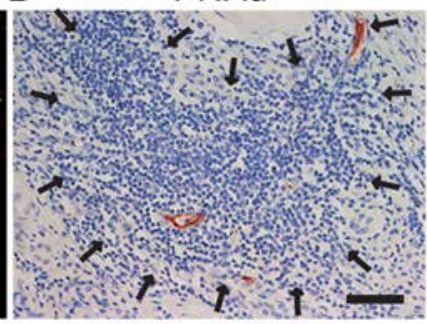

G $\quad \mathrm{CXCL} 13 / \mathrm{CD} 21$

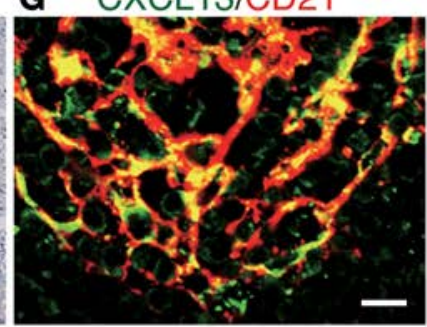

RALDH2

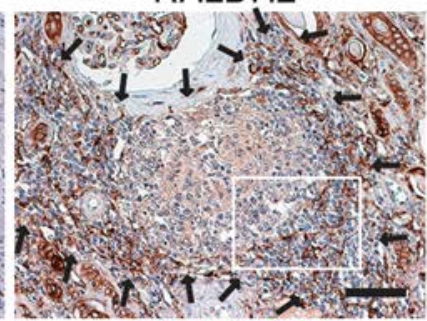

L p75NTR/CD21

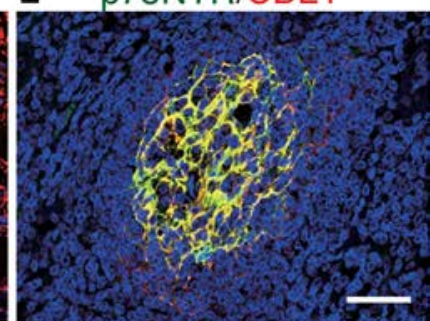

Figure 7. Tertiary lymphoid tissues (TLTs) form in aged human kidneys. (A-L) Histological analyses of aged human kidney samples. (A and B) Periodic acid-Schiff (PAS) staining and immunofluorescence analysis of (C) CD20 and CD3\&; (E) Ki67 and CD3e/CD20 (arrowheads indicate double-positive cells); (C) CXCL13 and CD21; (J) CXCL13 and CD45; (K) CD21, $\alpha$-smooth muscle actin ( $\alpha$ SMA), and CD45; and (L) p75 neurotrophin receptor (p75NTR) and CD21, and immunohistochemical analysis of (D) peripheral lymph node addressin (PNAd); (F) CD21 (arrowheads indicate the localization of $\mathrm{CD} 21^{+}$follicular dendritic cell [FDC] networks); (H) CD20, activation-induced cytidine deaminase (AID), CD21, and retinaldehyde dehydrogenase 2 (RALDH2) (serial sections); and (I) RALDH2 (arrowheads indicate the localization of $B$ cell follicles). The outlined region in $(\mathbf{H})$ is magnified in (I). Arrows indicate TLT localization. Scale bars: (A, D, H, K, and L) $50 \mu \mathrm{m},(\mathbf{B}, \mathbf{C}$, and F) $100 \mu \mathrm{m},(\mathbf{E}, \mathbf{G}, \mathbf{I}$, and J) $10 \mu \mathrm{m}$. (M) Quantitative analysis of TLT frequencies in human samples ( $n=56$; young $=13$, aged $=43$ ). ${ }^{\#} P<0.05$ (Pearson's $\chi^{2}$ test).

aggregates as TLTs. These TLTs also harbored a p75NTR ${ }^{+}$fibroblast network (Figure 5G) and CXCL13 and CCL19 ${ }^{+}$cells (Figure 5, H and I). In addition, the renal expression of Cxcl13, Ccl19, Ifng, and Tnfa was upregulated in an age-dependent manner (Figure 5J). Taken together, these data indicate that superaged mice spontaneously formed TLTs without injury, and that these were phenotypically equivalent to those in the aged injured kidney.

Targeting TLT formation has the potential to ameliorate renal fibrosis and inflammation. To assess the contribution of TLTs to long-term outcomes after kidney injury, we set up a series of experiments that could prevent the formation of renal TLTs after kidney injury in aged mice. First, we evaluated whether the loss of CXCL13 affects TLT formation. IRI-induced TLTs were smaller in aged CXCL13-deficient mice (Figure 6, A and B) than wild-type littermates, although TLTs had similar cellular components (Supplemental Figure 8). Furthermore, the expression of $C c 119$ and Ifng was significantly reduced in the CXCL13-deficient mice (Figure 6C). 
A

Fibroblast (p75NTR + / CCL19+)
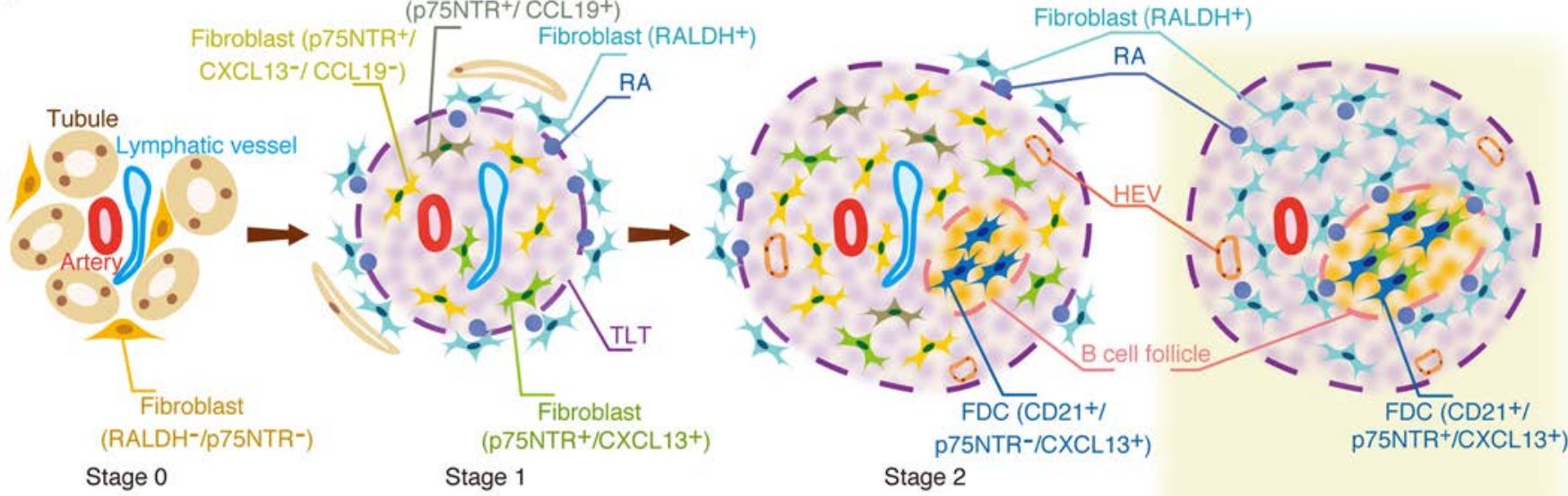
Stage 0 Stage 1 Stage 2

Mouse

Human

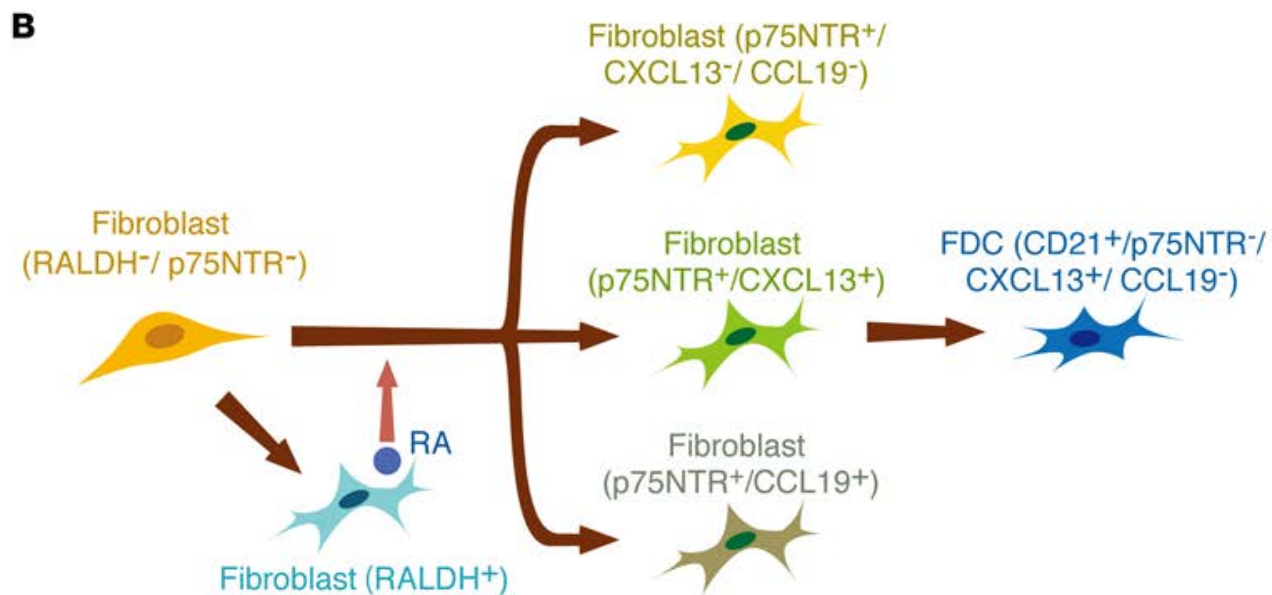

Figure 8. A model for renal tertiary lymphoid tissue (TLT) formation. Heterogeneous fibroblasts play crucial roles in steering the development and maturation of renal TLTs in mice and humans. (A) Mouse: In the early stage, fibroblasts around arteries receive retinoic acid (RA) from retinaldehyde dehydrogenase $2^{+}\left(\mathrm{RALDH}^{+}\right)$fibroblasts in a paracrine manner and dedifferentiate into p75 neurotrophin receptor ${ }^{+}\left(\right.$p75NTR $\left.^{+}\right)$fibroblasts, some of which acquire the ability to produce CXCL13/CCL19 (Stage 1). As the TLT grows and expands, the number of RA-producing fibroblasts around it gradually decreases (Stage 2). In this stage, CD21 ${ }^{+} /$p75NTR follicular dendritic cells (FDCs) emerge as a part of a stromal network, and peripheral lymph node addressin ${ }^{+}\left(\mathrm{PNAd}^{+}\right)$high endothelial venules (HEVs) develop within the TLT. Human: Although the major components in human renal TLTs are quite similar to those in mouse TLTs, p75NTR colocalizes with CD21, and RALDH ${ }^{+}$stromal cells surrounded these FDC networks. (B) A model of stromal cell maturation in the aged injured kidney. Upon kidney injury, resident fibroblasts differentiate into RALDH ${ }^{+}$fibroblasts, which promote the transdifferentiation of other fibroblasts into p75NTR+ fibroblasts with 3 phenotypes. These p75NTR+ fibroblasts act in concert to form TLTs. In the later phase of kidney injury, some of these fibroblasts lose their p75NTR expression and mature into CD21 ${ }^{+} /$CXCL13 ${ }^{+} /$p75NTR- FDCs.

Second, to test whether TLT formation depends on $\mathrm{CD} 4^{+}$cells, which are the major component of renal TLTs (Supplemental Figure 3A), we depleted $\mathrm{CD}^{+}$cells using the anti-CD4 monoclonal antibody GK1.5 (Figure 6D). We confirmed the depletion of $\mathrm{CD}^{+}$cells in the kidney and in the peripheral blood (Supplemental Figure 9). Mice treated with an isotype control antibody developed robust TLTs, whereas mice treated with GK1.5 did not develop TLTs (Figure 6, E and F), suggesting that TLT formation depended on $\mathrm{CD}^{+}$cells. Fibrosis score and the expression of homeostatic chemokines, proinflammatory cytokines, and fibrosis markers were significantly reduced in the GK1.5-treated kidneys (Figure 6, G and $\mathrm{H})$, suggesting that the deletion of TLTs could improve renal outcomes.

Third, we examined whether targeting the inflammatory microenvironment could suppress TLTassociated pathological conditions and improve renal outcomes. In most clinical situations, triggers of AKI, such as ischemic damage, arise without forewarning. We therefore administered dexamethasone (Dex) 2 weeks after ischemic insults (Figure 6I) and found that TLT formation was abolished and tubular injury was significantly attenuated in Dex-treated kidneys (Figure 6, J and K). Dex-treated mice exhibited a tendency to be less fibrotic than control mice, and the expression of homeostatic chemokines, pro- 
inflammatory cytokines, and fibrosis markers was also significantly reduced in Dex-treated mice (Figure 6, L and M), suggesting that antiinflammatory therapy could have the therapeutic potential to improve renal outcomes after AKI in the elderly by suppressing TLT-mediated inflammation.

TLTs form in aged human kidneys. Finally, we investigated whether our observations in mouse kidneys are relevant to human kidneys. In aged human kidneys, we found various degrees of chronic infiltrates around the arteries (Figure 7A), some of which harbored germinal center-like structures (Figure 7B). These human TLTs, like mouse TLTs, were composed mainly of T cells and B cells (Figure 7C) and contained HEVs (Figure 7D). Ki67 signals were also detectable within the aggregates (Figure 7E), suggesting that some of the chronic infiltrates functioned as TLTs. Some TLTs contained CD21 ${ }^{+}$FDC networks (Figure 7F) that were positive for CXCL13 (Figure 7G). Similar to our findings in mice, the CXCL13-positive cells were negative for CD45 (Figure 7J). Furthermore, AID was expressed within the B cell area in some TLTs (Figure 7H). As in mouse TLTs, fibroblasts outside the human renal TLTs had completely different phenotypes from those inside (Figure 7K). Unlike mouse renal TLTs, we found that p75NTR colocalized with CD21, an FDC marker, in human renal TLTs (Figure 7L), and $\mathrm{RALDH}^{+}$stromal cells surrounded these FDC networks (Figure 7, H and I), suggesting that, as in mouse renal TLTs, RA might play a crucial role in inducing p75NTR expression in human renal TLTs, especially in the development of FDC networks.

In specimens from 56 kidneys from patients (Supplemental Table 1), human TLTs, defined as inflammatory cell aggregates with CXCL13- and Ki67-positive signals inside, were significantly more prevalent in aged patients (over 60 years old, 28\%) than in young patients (under 40 years old, 0\%) (Figure 7M).

\section{Discussion}

Although epidemiological data established the relationship between AKI and subsequent CKD/ESRD $(1-3,5)$, there still has been no effective treatment and biomarker for the patients who progress to CKD/ ESRD after AKI. In the present study, we showed that, after kidney injury, TLTs developed within the kidney in an age-dependent manner and played pathogenic roles for the host in mouse and human. Our finding could lead to new treatment strategies and approaches to triage patients who have survived AKI, and potentially improve renal outcome in the aged AKI patients.

In this report, we defined TLTs as the organized lymphocyte aggregates composed of $\mathrm{T}$ cells and B cells with signs of proliferation. The definition of TLTs also includes the production of homeostatic chemokines such as CXCL13 and CCL19 inside TLTs. While HEVs and CD21 $1^{+}$FDCs were observed in larger TLTs, these findings were not included in the definition of TLTs, because small TLTs did not show these structures.

The developmental origin and phenotypic heterogeneity of stromal cells in TLTs have not been clarified due to the limitation of in vivo models $(25,26)$. Most in vivo TLT models are organ-specific transgenic mouse models ectopically expressing molecules stimulating TLT formation, which are artificial and incapable of fully mimicking the pathophysiology of TLTs. In the present study, we established a potentially novel model of inducible, sterile TLT formation, which has provided a solution to this limitation. This model and the genetic lineage-tracing method enabled us, for what we believe is the first time, to conduct a detailed analysis of the stromal cells underlying TLT formation, and we believe this is the first study that directly demonstrates the developmental origin of stromal cells, including FDCs, in TLTs.

The formation of TLTs in the inflamed organs has been associated with both protective and deleterious outcomes for the host (8-11). We demonstrated the direct destruction of neighboring parenchyma around renal TLTs, the close association between TLT sizes and impaired renal function, as well as renal expression of proinflammatory cytokines and AID, both of which support the notion that TLTs are detrimental to the aged injured kidney. Our findings indicate that the TLTs in aged kidneys are aberrant immunological foci, where pathogenic immune responses are generated. T cells, especially $\mathrm{CD} 4^{+} \mathrm{T}$ cells, were also observed around the TLTs, whereas most B cells were confined within the TLTs in the later phase in aged IRI kidneys (Supplemental Figure 10), suggesting that TLT-derived T cells might propagate inflammation across the entire kidney. In addition, the close association between TLT sizes and AID expression suggests that B cells might activate the molecular machinery responsible for producing affinity-maturated antibodies within the renal TLTs. Indeed, TLTs have been shown to support autoantibody production $(27,28)$, and the presence of TLTs predicts a poor outcome for chronic rejection $(29,30)$ and autoimmune disease (31). The presence of TLTs in the patients with IgA nephropathy, which is not age dependent, has also been correlated with poor renal outcomes (32). 
TLTs in the aged injured kidney exist in various stages of maturation (Figure 8A). In the early stage of TLT formation (Stage I), fibroblasts around arteries receive RA from $\mathrm{RALDH}^{+}$fibroblasts in a paracrine manner and dedifferentiate into p75NTR ${ }^{+}$fibroblasts, some of which acquire the ability to produce homeostatic chemokines (CXCL13/CCL19) (Figure 8, A and B). As the TLT grows and expands, the number of RA-producing fibroblasts around the TLT gradually decreases (Stage II). In this stage, CD21+ $75 \mathrm{NTR}^{-}$ FDCs emerge as a part of a stromal network, and peripheral lymph node addressin ${ }^{+}$(PNAd) $)^{+}$HEVs develop within the TLT. Importantly, TLTs were also observed in the aged but not young human kidneys, and the cellular and molecular components of these TLTs had strong similarities to mouse TLTs (Figure 8A). Thus, age-dependent renal TLT formation appears to be conserved across species, and the mechanism involved in the progression of human kidney disease in the elderly may be similar to that in aged mice. Notably, although renal TLTs have been reported only in patients with immune-medicated conditions, such as autoimmune disease, chronic rejection, glomerulonephritis, and infections $(29,32,33)$, we found age-dependent renal TLTs not only in the patients with the diseases above, but also in various types of kidney disease such as diabetic nephropathy and benign nephrosclerosis, indicating the universality of TLT formation with aging.

To define the cellular origin of TLT-associated fibroblasts described above, we performed a lineagetracing analysis utilizing P0-Cre mice, and for the first time to our knowledge, demonstrated that these TLT-associated heterogeneous fibroblasts, including FDCs, are derived from resident fibroblasts in the kidney (Figure 8B). This is consistent with a previous report that FDCs in the spleen arise from a subpopulation of PDGFR $\beta^{+}$resident stromal cells in the spleen after inflammatory stimuli (34). Our data not only add concrete evidence showing that FDCs originate from PDGFR $\beta^{+}$resident fibroblasts, but also suggest the developmental origin of FDCs through the lineage-tracing analysis with P0-Cre mice, which express Cre in the migrating neural crest and Schwann cells (22). Very recently, FDCs in the lymph node were reported to be lineage-labeled with Wnt-1 Cre (35), another Cre strain that labels neural crest-derived cells. Together with our findings in this study, these data suggest that, depending on the microenvironment, neural crest-derived cells have the potential to become homeostatic chemokineproducing fibroblasts, including FDCs. Of note, p75NTR ${ }^{+}$CCL19-producing fibroblasts inside TLTs, which may be the intermediate cellular elements capable of maturing into another stromal cell in TLTs, fibroblastic reticular cells (36), also simultaneously arise from $\mathrm{P0}$-Cre lineage-labeled resident fibroblasts.

We further demonstrated that a paracrine interaction between fibroblasts with distinct phenotypes triggers the initiation of TLT formation. The reciprocal expression pattern of RALDH and p75NTR in mouse and human TLTs (Figure 8A) and an in vitro experiment (Figure $3 \mathrm{H}$ ) indicates that fibroblast-derived RA promotes the expression of p75NTR in the fibroblasts inside TLTs to become the producer of CXCL13, whereas neuron-derived RA drives the expression of CXCL13 in lymphoid tissue organizer cells during the initiation of SLOs (16). To our knowledge, this is the first time that the involvement of RA has been demonstrated in the initiation of TLT formation.

The exclusive expression pattern of p75NTR in the fibroblasts inside TLTs led us to hypothesize that fibroblast phenotypes are determined by the microenvironment in which they reside. Notably, a novel lymphoid stromal cell type that can adapt its secretion of homeostatic chemokines according to its cellular environment was recently identified in the lymph node (37). Together, these data suggest that the phenotype of fibroblasts is plastic, and the regional microenvironments are critical determinants of their phenotype and could therefore be a therapeutic target.

Based on these observations, we conducted a series of interventional studies at molecular, cellular, and microenvironmental levels, to investigate the contribution of TLTs to disease progression in aged injured kidneys.

Smaller TLTs formed in aged CXCL13-deficient mice than in their wild-type littermates, suggesting that CXCL13 is crucial, although also redundant, for TLT formation in aged kidneys. The absence of lymph nodes in CXCL13-deficient mice (38) might also affect the severity of IRI, since lymph nodes play a crucial role in IRI pathophysiology (39). Further studies are required to determine whether CXCL13 is a suitable therapeutic target.

Furthermore, we found that depleting $\mathrm{CD}^{+}$cells abolished TLTs and resulted in the resolution of renal inflammation and fibrosis, suggesting that TLTs contribute to the sustained inflammation and impaired regeneration after AKI in the elderly. The significant contribution of $\mathrm{CD} 4^{+}$cells to TLT formation is consistent with previous reports in other TLT models (40-42). Among various types of immune cells, agedependent proportional and phenotypic changes of $\mathrm{CD}^{+} \mathrm{T}$ cells are most prominent, because of thymic 
involution with aging (24). Reduced thymic output results in lymphopenia, leading to a global phenotype shift from naive to memory $\mathrm{T}$ cells. Indeed, a bona fide, age-dependent, unique memory phenotype $\mathrm{CD} 4^{+} \mathrm{T}$ cell subpopulation was identified (43), which produces osteopontin and sclerostin domain-containing protein 1 (Sostdc1), also known as uterine sensitization-associated gene-1 (USAG-1), a novel BMP antagonist negatively regulating the renoprotective effect of BMP that we recently identified (44-47). The contribution of various types of $\mathrm{CD}^{+} \mathrm{T}$ cells to TLT formation should be analyzed in future studies.

Most importantly, we showed that administering Dex halted TLT formation and attenuated renal inflammation and fibrosis, even with late administration. Very recently, a single intraoperative, prophylactic treatment of high-dose Dex was found to effectively reduce the incidence of severe AKI after cardiac surgery in patients with advanced CKD (48); thus, Dex might also be a therapeutic option for AKI. Dex affects multiple types of cells and can dampen pathogenic immune responses directly or indirectly at multiple levels, although this amelioration is accompanied by side effects such as infections and diabetes.

Identifying patients at high risk for progressing to CKD/ESRD after AKI has the potential to reliably improve the treatment for these patients. Identifying the molecules involved in TLT formation will yield biomarkers with the requisite specificity to select groups of patients with TLTs and sustained inflammation. Implementation of such biomarker tests based on TLT-associated molecules may enable us to consider earlier clinical assessment, intervene in the patients at high risk for CKD/ESRD progression with currently available immunosuppressive agents such as Dex, and transfer patients to higher levels of care, therefore yielding improved outcomes. It is also important to acknowledge that future therapeutic strategies targeting the molecules and cell types specific for TLT formation might allow us to delete TLT more specifically and circumvent serious side effects of currently available immunosuppressive agents. Further studies are warranted to elucidate the molecular and cellular basis underlying TLT formation and establish biomarkers for selecting the patients developing TLTs, for translation of TLT-targeting therapies to human subjects.

Our data suggest a crucial role for RA in the initial step of TLT formation. While endogenous RA signaling was recently reported to have a reno-protective effect $(49,50)$, RA in the aged kidneys can trigger TLT formation, which promotes inflammation and hinders regeneration. Thus, RA's role in the injured kidney appears to be context dependent. Further understanding of the regulatory mechanism of RA production may therefore provide novel insight into adaptive and maladaptive repair after kidney injury.

Our study has limitations. First, neither the administration of GK1.5 nor Dex treatment is specific for TLT depletion. Depleting TLTs without affecting the entire immune system has been technically challenging because of the significant overlap in the mechanisms of SLO development and TLT formation, rendering the interpretation of the effects of TLT on the underlying disease difficult. In addition, it is still unclear why the renal environment becomes prone to TLT formation with aging.

In the present study, we shed light on the mechanisms underlying maladaptive repair in aged injured kidneys and identified previously unrecognized, age-dependent, pathogenic renal TLTs in mice and humans that have potential therapeutic relevance. Our findings indicate that TLTs could be a new target of therapeutic intervention for AKI in the elderly, and unraveling the cell types and molecules that govern TLT formation will open up a new avenue for overcoming kidney diseases in the elderly.

\section{Methods}

Animals. We selected 8-week-old, 12-month-old, and 23-month-old C57BL6J male mice as the young, aged, and superaged models, respectively, and purchased the mice from Japan SLC. P0-Cre (on an ICR background) mice (22) and R26ECFP (on a C57BL6 background) mice (51) were kindly provided by Kenichi Yamamura (Kumamoto University, Kumamoto, Japan) and Frank Costantini (Colombia University, New York, New York, USA), respectively. CXCL13-deficient mice (on a C57BL6 background) were described previously (52) and WT littermates were used as controls. Serum creatinine was measured by the creatininase-HMMPS method (SRL). All mice were maintained in specific pathogen-free conditions in the animal facility of Kyoto University. All animal experiments were approved by the Animal Research Committee, Graduate School of Medicine, Kyoto University, and were conducted in accordance with the Guide for the Care and Use of Laboratory Animals (NIH).

Kidney injury models. IRI, UUO, and FA nephropathy were induced as described previously (45, 46, 53). Briefly, IRI was induced by clamping unilateral renal pedicles for various lengths of time. Each group included at least 4 mice. In the FA nephropathy model, young $(n=12)$ and aged $(n=13)$ mice received FA (250 mg/kg; catalog F8758-25G; Sigma-Aldrich) in $0.15 \mathrm{M} \mathrm{NaHCO}_{3}$ on day 0 and were 
euthanized on day 21. Mice subjected to UUO were euthanized 14 days after the operations $(n=4$ in each group).

Renal histochemistry. Mouse kidneys were harvested, cut along the short axis at the maximum area of the whole kidney, fixed in Carnoy's solution, embedded in paraffin, sectioned (2.0- $\mu \mathrm{m}$ thickness), and then stained with periodic acid-Schiff (PAS) or periodic acid-methenamine-silver (PAM). Human kidneys were fixed in formalin, embedded in paraffin, sectioned (3.0- $\mu \mathrm{m}$ thickness), and stained with PAS.

Renal immunofluorescence. For immunofluorescence studies of mouse kidneys, the kidneys were fixed in $4 \%$ paraformaldehyde, incubated in 20\% sucrose for 6 hours, and incubated in $30 \%$ sucrose in PBS at $4^{\circ} \mathrm{C}$ overnight. OCT-embedded (Sakura Finetek) kidneys were cryosectioned into $6.0-\mu \mathrm{m}$ sections and mounted on Superfrost slides (Matsunami Glass). Sections were blocked with 5\% serum appropriate for the secondary antibody for 1 hour at room temperature, and then were incubated overnight at $4^{\circ} \mathrm{C}$ with primary antibodies. The following primary antibodies were used in the mouse immunohistochemistry and immunofluorescence experiments: anti- $\alpha$ SMA (catalog C6198; Sigma-Aldrich), -AID (catalog 4959; Cell Signaling), -GFP (catalog ab13970; Abcam), -Ki67 (catalog 14-5698; eBioscience), -CD45 (catalog 14-0451; eBioscience), -CD11c-PE (catalog 12-0114; eBioscience), -PDGFR- $\beta$ (catalog 14-1402; eBioscience), $-\beta 3$ tubulin-FITC (catalog A488-435L; Covance), -Cre (catalog 69050-3; Novagen), -LYVE-1 (catalog ab14917; Abcam), -CD21 (catalog ab75985; Abcam), -ER-TR7 (catalog T-2109; BMA Biomedicals), -CXCL13 (catalog AF470; R\&D Systems), -CCL19 (catalog AF880; R\&D Systems), -CCL21 (catalog AF457; R\&D Systems), - p75NTR (catalog AF1157; R\&D Systems), -CD3ع (catalog 550275; BD PharMingen), -B220 (catalog 557390; BD PharMingen), -PD-1 (catalog 114102; BioLegend), -CD4 (catalog 100726; BioLegend), -CD8 (catalog 100425; BioLegend), -PNAd (catalog 120801; BioLegend), and -ALDH1A2 (catalog HPA010022; Sigma-Aldrich) antibodies. ECFP was visualized with the anti-GFP antibody.

For immunofluorescence studies of human kidneys, kidney sections were fixed in formaldehyde and embedded in paraffin. The paraffin-embedded sections were deparaffinized with xylene, rehydrated, and then steam heated for 15 minutes. The sections were incubated with 5\% serum appropriate for the secondary antibody for 1 hour at room temperature, and then were incubated overnight at $4^{\circ} \mathrm{C}$ with primary antibodies as follows: anti-CD20 (catalog 14-0202; eBioscience), -CD45 (catalog 14-9457; eBioscience), -p75NTR (catalog AF1157; R\&D Systems), -PNAd (catalog 120801; BioLegend), -ALDH1A2 (catalog HPA010022; Sigma-Aldrich), -CD3 (catalog ab5690; Abcam), -CD21 (catalog ab75985; Abcam), -CXCL13 (catalog AF801; R\&D Systems), -Ki67 (catalog NCL-Ki67p; Vision BioSystems), and - $\alpha$ SMA (catalog C6198; Sigma-Aldrich) antibodies. Staining was visualized using the appropriate secondary antibodies. Slides were counterstained with DAPI. All of the immunofluorescence samples were analyzed with a confocal microscope (FV1000D; Olympus).

Renal immunohistochemistry. For immunohistochemistry, mouse and human kidneys were prepared as described above for the immunofluorescence studies of human kidneys. Endogenous peroxidase was blocked using 3\% $\mathrm{H}_{2} \mathrm{O}_{2}$. Antibody labeling was detected using a diaminobenzidine substrate kit (Vector Laboratories). Sections were counterstained with hematoxylin. The colocalization of CD20, CD21, and AID was determined by the staining of serial sections. All of the immunohistochemistry samples were analyzed with a Zeiss Axio Imager A2 microscope using Zeiss Axio Vision 4.8 software.

Quantitative analysis for renal fibrosis. For the quantitative analysis of renal fibrosis, the fibrotic area in Masson trichrome-stained sections was quantified using Adobe Photoshop software (Adobe) by determining the number of pixels present in the interstitial fibrotic area. The average ratios of fibrotic area to each microscopic field from the 8 images was calculated for each sample and expressed as the percentage area.

Quantification of TLT size. The renal TLT sizes were examined in PAS-stained sections of injured kidneys. TLT size was defined as the total cumulative size of the TLTs in the renal cortex of the sample. Pictures that included TLTs were taken at the same size and resolution, and TLT size was measured by an experienced renal pathologist using Adobe Photoshop software.

Real-time RT-PCR analysis. RNA extraction and real-time RT-PCR were performed as described previously $(45,46)$. The primer sequences are listed in Supplemental Table 2. Expression levels were normalized to those of Gapdh and expressed relative to levels in the young mouse kidney on day 0 (IRI) (Figures 1, 2, 3I, and 5), aged mouse kidney on day 0 (IRI) (Figure 6), or in controls (Figure 3H).

GK1.5 administration. GK1.5 (catalog 40-0041; Tonbo Biosciences) $(150 \mu \mathrm{g}, n=5)$ or isotype-matched control immunoglobulin G (catalog 400640; clone RTK4530; BioLegend) (150 $\mu \mathrm{g}, n=6)$ was administered to aged mice by intraperitoneal injection 2 days before and at 8,22 , and 35 days after unilateral 
30-minute IRI. Mice were euthanized 45 days after IRI. The efficacy of CD4 ${ }^{+}$cell depletion was confirmed by flow cytometric analysis of peripheral blood cells and by immunostaining for CD4 in kidney samples harvested on day 45.

Dex administration. Mice underwent 30-minute IRI on day 0 and received $8 \mathrm{mg} / \mathrm{kg}$ Dex $(\mathrm{MSD})(n=5)$ or PBS $(n=5)$ intraperitoneally on days 14,15 , and 16 . On day 17 , an osmotic minipump (model 2001; Alzet) with either Dex (79.2 $\mu \mathrm{g} /$ day) or PBS vehicle was placed in a subcutaneous pouch. Mice were euthanized 24 days after IRI.

Flow cytometric analysis. Red blood cells were lysed with ACK lysis buffer $\left(0.15 \mathrm{M} \mathrm{NH}_{4} \mathrm{Cl}, 10 \mathrm{mM}\right.$ $\mathrm{KHCO}_{3}, 0.1 \mathrm{mM} \mathrm{Na}$ EDTA, $\mathrm{pH}$ 7.2-7.4), and the remaining single-cell suspensions were blocked with anti-CD16/32 antibody (clone 2.4G2) and then stained with PE-conjugated anti-CD3e (catalog 15-0031-83; eBioscience), FITC-conjugated anti-TCR $\beta$ (catalog 109206; BioLegend), PE-Cy7-conjugated anti-CD4 (catalog 60-0041-U100; Tonbo Bioscience), and APC-conjugated anti-CD8 antibodies (catalog 20-1886-U100; Tonbo Bioscience). All flow cytometric analyses were performed using a FACSCanto (BD Bioscience).

Cell culture experiments. C3H10T1/2 cells were purchased from the Health Science Research Sources Bank (Osaka, Japan). Renal PDGFR $\beta^{+}$fibroblasts were sorted as described previously (19), using a PE-conjugated anti-PDGFR $\beta$ antibody (catalog 12-1402; eBioscience). Both cell types were cultured with DMEM supplemented with 10\% FCS and were treated with vehicle (ethanol) or with $25 \mathrm{nM}$ or $50 \mathrm{nM}$ RA (catalog R2625-50MG; Sigma-Aldrich) for 9 hours.

Analysis of human kidney specimens. All of the human specimens were procured and analyzed after informed consent and with approval of the Ethics Committee of Kyoto University Hospital. Kidneys from nephrectomy cases for renal cell carcinoma and from autopsy cases in Kyoto University Hospital were analyzed. Experienced pathologists confirmed that all of the nephrectomy samples analyzed in this study were free of carcinoma. For the quantitative analysis of TLT formation frequencies, kidneys from 47 nephrectomy cases for renal cell carcinoma and from 9 autopsy cases in Kyoto University Hospital were analyzed. Because TLTs can develop as a result of certain diseases, patients who had a clinical history and/ or laboratory evidence of the following diseases were excluded: (a) pyelonephritis, (b) glomerulonephritis, (c) autoimmune kidney disease, and (d) hematological malignancies. We defined young patients as those 40 years of age and under, and aged patients as those 60 and over. Clinical data including medical history, complications, and laboratory data are detailed in Supplemental Table 1. To discriminate between chronic infiltrates and TLTs in human samples, the TLTs were defined as mononuclear perivascular cell aggregates that contained both CXCL13 and Ki67 signals. An experienced renal pathologist assessed the frequency of TLTs in each specimen.

Statistics. Data are reported as dot plots with the average \pm SD or as box and whisker plots. Statistical significance was assessed by a 2-tailed Student's $t$ test for comparisons between 2 groups, 1-way ANOVA with Tukey's post-hoc tests for comparisons among more than 2 groups, and Pearson's $\chi^{2}$ test in Figure $7 \mathrm{M}$ and Supplemental Table 1. Correlations were determined by Pearson's correlation analysis. Statistical analyses were performed using JMP software (version 9.0). For all analyses, we considered $P$ values less than 0.05 to be statistically significant.

Study approval. All animal experiments were approved by the Animal Research Committee, Graduate School of Medicine, Kyoto University, and were conducted in accordance with the Guide for the Care and Use of Laboratory Animals (NIH). All of the human specimens were procured and analyzed after informed consent and with approval of the ethical committee of Kyoto University Hospital.

\section{Author contributions}

YS and MoY designed the experiments and wrote the manuscript. MoY supervised the project. YS performed experiments and collected and analyzed data. AM, HF, HN, KM, TK, and MA performed some of the experiments and collected data. SNi, SS, HH, and $\mathrm{OO}$ helped collect human samples and data. YH, AS, SNa, MaY, MM, KS, NM, and HK discussed the results and commented on the manuscript.

\section{Acknowledgments}

We are grateful to Tasuku Honjo, Shigekazu Nagata, Toru Nakano, and Takashi Nagasawa for their valuable comments and discussions. We are also greatly appreciative of Ken-ichi Yamamura for providing the P0-Cre mice, and of Frank Costantini for providing the R26ECFP mice. We also thank Tatsuji Haga, 
Yumiko Tomita, Chiyomi Inoue, Ayaka Morita, and Maki Ozone for their excellent technical assistance. This study was supported by Grant-in-Aid for Scientific Research B (26293202) from the Japan Society for the Promotion of Science (JSPS), CREST from the Japan Science and Technology Agency, and partly by the grants from the TMK Project, Mitsubishi Tanabe Pharma Corporation, Uehara Memorial Foundation, Takeda Science Foundation, and the Sumitomo Foundation.

Address correspondence to: Motoko Yanagita, Department of Nephrology, Graduate School of Medicine, Kyoto University, Shogoin-Kawahara-cho 54, Sakyo-ku, Kyoto 606-8507, Japan. Phone: 81.75.751.3860; E-mail: motoy@kuhp.kyoto-u.ac.jp.

Yuki Sato's present address is: TMK Project, Graduate School of Medicine, Kyoto University, Kyoto, Japan. Akiko Mii's present address is: Department of Nephrology, Graduate School of Medicine, Nippon Medical School, Tokyo, Japan. Harumi Fujita's present address is Department of Dermatology, Keio University School of Medicine, Tokyo, Japan.

1. Lewington AJ, Cerdá J, Mehta RL. Raising awareness of acute kidney injury: a global perspective of a silent killer. Kidney Int 2013;84(3):457-467.

2. Bonventre JV, Yang L. Cellular pathophysiology of ischemic acute kidney injury. J Clin Invest. 2011;121(11):4210-4221.

3. Molitoris BA. Therapeutic translation in acute kidney injury: the epithelial/endothelial axis. J Clin Invest. 2014;124(6):23552363.

4. Hsu RK, McCulloch CE, Dudley RA, Lo LJ, Hsu CY. Temporal changes in incidence of dialysis-requiring AKI. J Am Soc Nephrol. 2013;24(1):37-42.

5. Ishani A, et al. Acute kidney injury increases risk of ESRD among elderly. J Am Soc Nephrol. 2009;20(1):223-228.

6. Medzhitov R. Origin and physiological roles of inflammation. Nature. 2008;454(7203):428-435.

7. Rabb H, et al. Inflammation in AKI: current understanding, key questions, and knowledge gaps. J Am Soc Nephrol. 2016;27(2):371-379.

8. Neyt K, Perros F, GeurtsvanKessel CH, Hammad H, Lambrecht BN. Tertiary lymphoid organs in infection and autoimmunity. Trends Immunol. 2012;33(6):297-305.

9. Pitzalis C, Jones GW, Bombardieri M, Jones SA. Ectopic lymphoid-like structures in infection, cancer and autoimmunity. Nat Rev Immunol. 2014;14(7):447-462.

10. van de Pavert SA, Mebius RE. New insights into the development of lymphoid tissues. Nat Rev Immunol. 2010;10(9):664-674.

11. Ruddle NH. Lymphatic vessels and tertiary lymphoid organs. J Clin Invest. 2014;124(3):953-959.

12. Moyron-Quiroz JE, et al. Role of inducible bronchus associated lymphoid tissue (iBALT) in respiratory immunity. Nat Med. 2004;10(9):927-934.

13. Lee Y, et al. Recruitment and activation of naive T cells in the islets by lymphotoxin beta receptor-dependent tertiary lymphoid structure. Immunity. 2006;25(3):499-509.

14. Okazaki T, Chikuma S, Iwai Y, Fagarasan S, Honjo T. A rheostat for immune responses: the unique properties of PD-1 and their advantages for clinical application. Nat Immunol. 2013;14(12):1212-1218.

15. Ishida Y, Agata Y, Shibahara K, Honjo T. Induced expression of PD-1, a novel member of the immunoglobulin gene superfamily, upon programmed cell death. EMBO J. 1992;11(11):3887-3895.

16. van de Pavert SA, et al. Chemokine CXCL13 is essential for lymph node initiation and is induced by retinoic acid and neuronal stimulation. Nat Immunol. 2009;10(11):1193-1199.

17. Blomhoff R, Blomhoff HK. Overview of retinoid metabolism and function. J Neurobiol. 2006;66(7):606-630.

18. Nagoshi N, Shibata S, Nakamura M, Matsuzaki Y, Toyama Y, Okano H. Neural crest-derived stem cells display a wide variety of characteristics. J Cell Biochem. 2009;107(6):1046-1052.

19. Asada N, et al. Dysfunction of fibroblasts of extrarenal origin underlies renal fibrosis and renal anemia in mice. J Clin Invest. 2011;121(10):3981-3990.

20. Heesters BA, Myers RC, Carroll MC. Follicular dendritic cells: dynamic antigen libraries. Nat Rev Immunol. 2014;14(7):495-504

21. Muramatsu M, Kinoshita K, Fagarasan S, Yamada S, Shinkai Y, Honjo T. Class switch recombination and hypermutation require activation-induced cytidine deaminase (AID), a potential RNA editing enzyme. Cell. 2000;102(5):553-563.

22. Yamauchi Y, et al. A novel transgenic technique that allows specific marking of the neural crest cell lineage in mice. Dev Biol. 1999;212(1):191-203.

23. Duffield JS. Cellular and molecular mechanisms in kidney fibrosis. J Clin Invest. 2014;124(6):2299-2306.

24. Goronzy JJ, Weyand CM. Understanding immunosenescence to improve responses to vaccines. Nat Immunol. 2013;14(5):428-436

25. Aloisi F, Pujol-Borrell R. Lymphoid neogenesis in chronic inflammatory diseases. Nat Rev Immunol. 2006;6(3):205-217.

26. Kain MJ, Owens BM. Stromal cell regulation of homeostatic and inflammatory lymphoid organogenesis. Immunology. 2013;140(1):12-21.

27. Humby F, et al. Ectopic lymphoid structures support ongoing production of class-switched autoantibodies in rheumatoid synovium. PLoS Med. 2009;6(1):e1.

28. Bombardieri M, et al. Activation-induced cytidine deaminase expression in follicular dendritic cell networks and interfollicular large B cells supports functionality of ectopic lymphoid neogenesis in autoimmune sialoadenitis and MALT lymphoma in Sjögren's syndrome. J Immunol. 2007;179(7):4929-4938. 
29. Thaunat O, et al. Lymphoid neogenesis in chronic rejection: evidence for a local humoral alloimmune response. Proc Natl Acad Sci USA. 2005;102(41):14723-14728.

30. Thaunat $\mathrm{O}$, et al. Chronic rejection triggers the development of an aggressive intragraft immune response through recapitulation of lymphoid organogenesis. J Immunol. 2010;185(1):717-728.

31. Drayton DL, Liao S, Mounzer RH, Ruddle NH. Lymphoid organ development: from ontogeny to neogenesis. Nat Immunol. 2006;7(4):344-353.

32. Pei G, et al. Renal interstitial infiltration and tertiary lymphoid organ neogenesis in IgA nephropathy. Clin J Am Soc Nephrol. 2014;9(2):255-264.

33. Steinmetz OM, et al. Analysis and classification of B-cell infiltrates in lupus and ANCA-associated nephritis. Kidney Int. 2008;74(4):448-457.

34. Krautler NJ, et al. Follicular dendritic cells emerge from ubiquitous perivascular precursors. Cell. 2012;150(1):194-206

35. Jarjour M, et al. Fate mapping reveals origin and dynamics of lymph node follicular dendritic cells. J Exp Med. 2014;211(6):1109-1122.

36. Fletcher AL, Acton SE, Knoblich K. Lymph node fibroblastic reticular cells in health and disease. Nat Rev Immunol. 2015;15(6):350-361.

37. Mionnet C, et al. Identification of a new stromal cell type involved in the regulation of inflamed B cell follicles. PLoS Biol. 2013;11(10):e1001672

38. Ansel KM, et al. A chemokine-driven positive feedback loop organizes lymphoid follicles. Nature. 2000;406(6793):309-314.

39. Dong X, Swaminathan S, Bachman LA, Croatt AJ, Nath KA, Griffin MD. Antigen presentation by dendritic cells in renal lymph nodes is linked to systemic and local injury to the kidney. Kidney Int. 2005;68(3):1096-1108.

40. Rangel-Moreno J, et al. The development of inducible bronchus-associated lymphoid tissue depends on IL-17. Nat Immunol. 2011;12(7):639-646

41. Peters A, et al. Th17 cells induce ectopic lymphoid follicles in central nervous system tissue inflammation. Immunity. 2011;35(6):986-996

42. Marinkovic $\mathrm{T}$, et al. Interaction of mature $\mathrm{CD} 3{ }^{+} \mathrm{CD} 4^{+} \mathrm{T}$ cells with dendritic cells triggers the development of tertiary lymphoid structures in the thyroid. J Clin Invest. 2006;116(10):2622-2632.

43. Shimatani K, Nakashima Y, Hattori M, Hamazaki Y, Minato N. PD-1+ memory phenotype CD4+ T cells expressing C/EBPalpha underlie T cell immunodepression in senescence and leukemia. Proc Natl Acad Sci USA. 2009;106(37):15807-15812.

44. Yanagita M, et al. USAG-1: a bone morphogenetic protein antagonist abundantly expressed in the kidney. Biochem Biophys Res Commun. 2004;316(2):490-500.

45. Yanagita M, et al. Uterine sensitization-associated gene-1 (USAG-1), a novel BMP antagonist expressed in the kidney, accelerates tubular injury. J Clin Invest. 2006;116(1):70-79.

46. Tanaka M, et al. Expression of BMP-7 and USAG-1 (a BMP antagonist) in kidney development and injury. Kidney Int. 2008;73(2):181-191

47. Tanaka M, et al. Loss of the BMP antagonist USAG-1 ameliorates disease in a mouse model of the progressive hereditary kidney disease Alport syndrome. J Clin Invest. 2010;120(3):768-777.

48. Jacob KA, et al. Intraoperative high-dose dexamethasone and severe AKI after cardiac surgery. J Am Soc Nephrol. 2015;26(12):2947-2951.

49. Peired A, et al. Proteinuria impairs podocyte regeneration by sequestering retinoic acid. J Am Soc Nephrol. 2013;24(11):17561768.

50. Chiba T, et al. Retinoic acid signaling coordinates macrophage-dependent injury and repair after AKI. J Am Soc Nephrol. 2016;27(2):495-508.

51. Srinivas S, et al. Cre reporter strains produced by targeted insertion of EYFP and ECFP into the ROSA26 locus. BMC Dev Biol. 2001;1:4.

52. Ebisuno Y, et al. Cutting edge: the B cell chemokine CXC chemokine ligand 13/B lymphocyte chemoattractant is expressed in the high endothelial venules of lymph nodes and Peyer's patches and affects B cell trafficking across high endothelial venules. J Immunol. 2003;171(4):1642-1646.

53. Endo T, et al. Exploring the origin and limitations of kidney regeneration. J Pathol. 2015;236(2):251-263. 